\title{
GMPE-Consistent \\ Hard-Rock Site Adjustment Factors for Western North \\ America
}

2 Linda Al Atik, ${ }^{1}$ M.EERI, Nicholas J. Gregor, ${ }^{2}$ M.EERI, Norman A.

${ }_{3}$ Abrahamson, ${ }^{3}$ Albert R. Kottke, ${ }^{4}$ M.EERI, 


\begin{abstract}
Empirical ground-motion prediction equations [GMPEs] such as the Next Generation Attenuation-West2 [NGA-West2] GMPEs are limited in the number of recordings on hard-rock stations used to develop the models. Therefore, the site response scaling in the GMPEs cannot be reliably extrapolated to hard-rock conditions. The state of practice for the development of hard-rock adjustment factors involves the use of analytical methods that typically assign small values to the high-frequency small strain damping $\left[\kappa_{0}\right]$ for hard-rock sites resulting in large scaling factors at short periods. Alternatively, the hard-rock scaling factors developed in Ktenidou and Abrahamson (2016) [KA16] based on empirical groundmotion data are used. These empirical factors, developed for a broad rock site 4 category, show that the average hard-rock scaling factors observed in groundmotion data are small in amplitude.

To address the shortcomings in the current state of practice, we present a methodology to develop hard-rock linear site adjustment factors to adjust the NGAWest2 GMPEs from $V_{S 30}$ of $760 \mathrm{~m} / \mathrm{sec}$ to target hard-rock site conditions with $V_{S 30}$ ranging from 1000 to $2200 \mathrm{~m} / \mathrm{sec}$. These factors are analytically derived using the IRVT approach of Al Atik et al. (2014) but with inputs constrained using the empirical KA16 factors and normalized to the scaling of the NGA-West2 GMPEs for $V_{S 30}$ of $1000 \mathrm{~m} / \mathrm{sec}$. The proposed factors merge the results of the NGA-West2 site response scaling for $V_{S 30} \leq 1000 \mathrm{~m} / \mathrm{sec}$ with the KA16 hard-rock category factors to produce a site factor model that is a continuous function of $V_{S 30}$. The epistemic uncertainty of these factors is evaluated.
\end{abstract}

\title{
Keywords
}

5 Site response, site adjustment, site amplification, $\kappa_{0}$, hard rock, GMPE consistent, NGA-West2

\footnotetext{
${ }^{1}$ Linda Alatik Consulting, San Francisco, CA

${ }^{2}$ Consultant, Oakland, CA

${ }^{3}$ University of California, Berkeley, CA

${ }^{4}$ Pacific Gas \& Electric, San Francisco, CA
}

Corresponding author:

Linda Al Atik, San Francisco, CA

Email: linda.alatik@gmail.com

Prepared using sagej.cls [Version: 2017/01/17 v1.20] 


\section{Introduction}

Modern ground-motion prediction equations [GMPEs] such as the Next Generation Attenuation-West2 [NGA-West2] GMPEs characterize site response as a continuous function of the time-averaged shear-wave velocity over the top $30 \mathrm{~m}$ of the site profile $\left[V_{S 30}\right]$. Other parameters, such as the depth to a shear-wave velocity of 1.0 or $2.5 \mathrm{~km} / \mathrm{s}$ horizon [ $Z_{1.0}$ and $Z_{2.5}$ ], are used to characterize the long-period site amplification due to basin effects. Nonlinear site response is modeled in the GMPEs as a function of $V_{S 30}$ and the median spectral acceleration or peak ground acceleration on rock. The histogram of the number of recording stations in the different $V_{S 30}$ bins used in the development of the Abrahamson et al. (2014) GMPE [ASK14] is shown in Figure 1. This figure shows that the number of recording stations with $V_{S 30}>1000 \mathrm{~m} / \mathrm{sec}$ is limited. The $V_{S 30}$ dependence of the site factors is modeled in the GMPEs as a linear function of $\ln \left(V_{S 30}\right)$. With the sampling of $V_{S 30}$ in the NGA-West2 dataset, the coefficient for $\ln \left(V_{S 30}\right)$ is constrained by empirical ground-motion data for $V_{S 30}$ values between 200 and $800 \mathrm{~m} / \mathrm{s}$. For hard-rock sites with $V_{S 30}>1000 \mathrm{~m} / \mathrm{sec}$, the site factor is based on an extrapolation of this slope to high $V_{S 30}$ values with little empirical constraints. To reflect the limited hard-rock data, some GMPEs limit the reduction of the site factor at high $V_{S 30}$ values (e.g. $1500 \mathrm{~m} / \mathrm{s}$ ).

Empirical hard-rock adjustment factors were developed in Ktenidou and Abrahamson (2016) [KA16] to adjust GMPEs from $V_{S 30}$ of $760 \mathrm{~m} / \mathrm{sec}$ to average hard-rock conditions based on the Next Generation Attenuation-East Project [NGAEast] and the BCHydro British Columbia ground-motion datasets. These factors were developed using the average of total ground-motion residuals with $V_{S 30}$ greater than $1000 \mathrm{~m} / \mathrm{sec}$ relative to median predictions from an interim NGA-East GMPE and the Chiou and Youngs (2014) [CY14] GMPE with $V_{S 30}$ of $760 \mathrm{~m} / \mathrm{sec}$ for the NGA-East and the BCHydro datasets, respectively. These scaling factors account for differences in the $V_{S}$ profiles and the high-frequency small-strain damping $\left[\kappa_{0}\right]$ between the reference site condition in the GMPEs with $V_{S 30}$ of $760 \mathrm{~m} / \mathrm{sec}$ and an average hard-rock site in the NGA-East and the BCHydro datasets. Figure 2 presents the two hard-rock scaling models proposed by KA16: model 2 is based on the residual analysis of the BCHydro dataset and model 1 is based on an average of the scaling obtained for the combined BCHydro and the NGA-East datasets. KA16 indicate that the average $V_{S 30}$ for the BCHydro and the NGA-East data used to develop the models is 2380 and 1975 m/sec, respectively. The KA16 models shown in Figure 2 were developed as an interim measure to provide an alternative to the typically large scale factors computed

Prepared using sagej.cls 
using analytical methods for hard-rock sites. These models show that observed groundmotion data on hard-rock sites, on average, do not show the large scale factors at short periods typically found in analytical studies that assign small $\kappa_{0}$ values, on the order of $0.006 \mathrm{sec}$, for hard-rock sites.

The KA16 hard-rock adjustment factors suffer from shortcomings stemming from the poor characterization of site conditions at the recordings stations in the NGAEast and BCHydro datasets and the low sample rates at some seismic stations that limit the useable frequency band. Most of the hard-rock stations in the NGA-East dataset do not have measured $V_{S 30}$ values, and the NGA-East ground motions suffer from frequency-bandwidth limitations affecting data quality at the low and the high frequencies. Similarly, all rock stations in the BCHydro dataset are classified based on surface geology (Ktenidou and Abrahamson 2016). Errors in the $V_{S 30}$ estimates for the hard-rock sites could lead to misclassification of the sites and affect the resulting scale factors and average $V_{S 30}$ values. Another limitation of the KA16 factors is that they are not a continuous function of $V_{S 30}$ and instead apply to a broad hard-rock site class with $V_{S 30}>1000 \mathrm{~m} / \mathrm{sec}$. This is a result of the limited recordings on hard-rock sites in the empirical datasets.

The current state of practice for the development of site adjustment factors for hardrock site conditions involves three different approaches: (1) use of $V_{S}-\kappa_{0}$ correction factors developed using analytical methods with assigned target $\kappa_{0}$ values and $V_{S}$ profiles; (2) use of empirical hard-rock factors such as the KA16 factors which are developed for a broad category of hard-rock sites; and (3) use of the $V_{S 30}$ scaling in the NGA-West2 GMPEs extrapolated to hard-rock $V_{S 30}$ despite the limited empirical data on hard-rock site conditions available to constrain the $V_{30}$ scaling and the lack of explicit $\kappa_{0}$ scaling in the GMPEs. To address the shortcomings in the current state of practice, we present a methodology to develop hard-rock site factors to adjust the NGA-West2 GMPEs from $V_{S 30}$ of $760 \mathrm{~m} / \mathrm{sec}$ to target hard-rock site conditions with $V_{S 30}$ ranging from 1000 to $2200 \mathrm{~m} / \mathrm{sec}$. These factors are analytically derived using the IRVT approach of Al Atik et al. (2014) but constrained using the empirical KA16 factors and also normalized to the NGA-West 2 site factors for $V_{S 30}$ of $1000 \mathrm{~m} / \mathrm{sec}$. These empirical constraints allow for the calibration of the hard-rock properties in the analytical study so that they are consistent with the observed ground-motion scaling for these sites. The proposed factors merge the results of the NGA-West $2 V_{S 30}$ scaling for $V_{S 30} \leq 1000 \mathrm{~m} / \mathrm{sec}$ with the KA16 hard-rock category factors to produce a model for the site factors that is a continuous function of $V_{S 30}$ and consistent with the empirical hard-rock factors.

Prepared using sagej.cls 
Our approach starts with an evaluation of the average hard-rock site conditions representative of the KA16 scaling factors, by inverting for the average $V_{S 30}, V_{S}$ profile, and $\kappa_{0}$ implicit in the KA16 models. Next, the target site conditions $\left(V_{S}(z)\right.$ profile and $\kappa_{0}$ ) are defined for a range of hard-rock site conditions with $V_{S 30}$ of 1000 to $2200 \mathrm{~m} / \mathrm{sec}$ based on a literature review of hard-rock $V_{S}$ profiles and $\kappa_{0}$ estimates in Western North America [WNA]. Target site parameters are adjusted using empirical constraints, and hard-rock site adjustment factors are derived and presented for a suite of thirteen target $V_{S 30}$ values between 1000 and $2200 \mathrm{~m} / \mathrm{sec}$.

The site factors presented in this paper extend the NGA-West2 linear site response scaling to hard-rock site conditions with target hard-rock sites defined based on WNA average rock site properties. Therefore, our derived hard-rock site adjustment factors are applicable for target sites in WNA. We use the KA16 models derived from empirical NGA-East and BCHydro data as constraints because of the scarcity of empirical ground-motion data on hard rock sites in WNA. Moreover, KA16 showed that average hard-rock scaling outside of WNA is not drastically different than what would be expected for WNA sites. We note that the hard-rock factors presented in this paper are intended for use at sites with limited site characterization, such as sites with measured or estimated $V_{S 30}$ values, but without measured $V_{S}(z)$ profiles. For hard-rock sites with measured $V_{S}(z)$ profiles, site-specific hard-rock adjustment factors should be computed based on the site profile with its appropriate epistemic uncertainty.

\section{Vs Profile and $\kappa_{0}$ Inversion for the KA16 Models}

The use of the KA16 models to constrain the analytical hard-rock site adjustment factors requires an evaluation of the implied average site conditions representative of these scaling factors. Because a large number of the stations used in the KA16 analysis did not have measured $V_{S 30}$ and $\kappa_{0}$ values, we use the $\kappa_{0}$ and $V_{S}$ profile inversion methodology of Al Atik and Abrahamson (2021) to invert for representative $V_{S 30}$, $V_{S}(z)$ profile, and $\kappa_{0}$ for the average site conditions implied by the KA16 hard-rock scaling factors.

The inversion is performed using the CY14 GMPE because KA16 model 2 is based on the residual analysis of the BCHydro dataset with respect to CY14 and because the spectral shape of the CY14 GMPE generally falls in the center of the range of spectral shapes from the NGA-West2 GMPEs. The first step involves converting the KA16 hard-rock scaling factors from pseudo-spectral acceleration [PSA] domain to Fourier amplitude spectra [FAS] domain. As such, CY14 median response spectra for strike-slip scenarios with magnitude 5, 6, and 7, distance of 5, 10, and $20 \mathrm{~km}$ and 
$V_{S 30}$ of $760 \mathrm{~m} / \mathrm{sec}$ are computed. These scenarios are selected to capture the shortdistance $\kappa_{0}$ scaling from a range of hazard-significant magnitudes. The CY14 median response spectra are corrected to hard-rock conditions by multiplying them with the KA16 model 1 and model 2 factors. Next, the IRVT approach of Al Atik et al. (2014) is used to convert the GMPE's response spectra for $V_{S 30}=760 \mathrm{~m} / \mathrm{sec}$ and the spectra corrected to hard-rock conditions into corresponding FAS. Duration estimates for the different scenarios are calculated using estimates of source and path durations with generic Western US [WUS] parameters based on Campbell (2003). The peak factor of Vanmarke (1975) is used in the IRVT method. The FAS for the scenarios with $V_{S 30}=$ $760 \mathrm{~m} / \mathrm{sec}$ and those corrected to hard-rock conditions are presented in Figure 3. These IRVT-based FAS show a change in their spectral shape at frequency of about $50 \mathrm{~Hz}$. This is likely due to saturation effects in the IRVT process discussed in Al Atik et al. (2014). For the hard-rock FAS, the sharp change observed around $50 \mathrm{~Hz}$ is caused by the sharp changes in the KA16 factors in the same frequency range, particularly for KA16 model 2.

For each earthquake scenario, the ratio of FAS for the hard-rock site condition relative to FAS for $V_{S 30}=760 \mathrm{~m} / \mathrm{sec}$ is computed. Figure 4 presents these ratios for each of the nine scenarios considered for model 1 and model 2 along with the average of the ratios over the nine scenarios. These ratios approximate the FAS linear site factors for hard rock relative to the reference site condition with $V_{S 30}$ of $760 \mathrm{~m} / \mathrm{sec}$. These relative site factors represent the differences in the $V_{S}$ profile and $\kappa_{0}$ scaling between the average hard-rock site implied by the KA16 models and the average site condition for CY14 for $V_{S 30}$ of $760 \mathrm{~m} / \mathrm{sec}$. The FAS ratios are stable over all nine scenarios for frequencies up to about $20-30 \mathrm{~Hz}$ as shown in Figure 4. For frequencies greater than $20 \mathrm{~Hz}$, the FAS ratios start diverging due to potential saturation effects in the IRVTderived FAS discussed in Al Atik et al. (2014). The average relative site factors are smoothed as shown in Figure 5. We note that these average relative FAS site factors are considered reliable for frequencies between 0.6 than $30 \mathrm{~Hz}$. The upper limit is imposed to avoid potential saturation effects in the IRVT-based FAS and the lower limit is based on the KA16 factors being constrained by data for frequencies up to $0.6 \mathrm{~Hz}$.

The next step involves converting the hard-rock site factors relative to $760 \mathrm{~m} / \mathrm{sec}$ to total site factors (relative to the $V_{S}$ and density at the source depth). As such, we use the CY14-compatible $V_{S}$ profile and $\kappa_{0}$ of Al Atik and Abrahamson (2021) as representative of the reference site condition with $V_{S 30}$ of $760 \mathrm{~m} / \mathrm{sec}$. The reference $V_{S}$ profile and corresponding quarter-wavelength [QWL] linear site amplification for CY14 for $V_{S 30}$ of $760 \mathrm{~m} / \mathrm{sec}$ are shown in Figure 10. The QWL site amplification is computed according to Boore (2003) with a zero angle of incidence and source $V_{S}$ and

Prepared using sagej.cls 
density set at $3.5 \mathrm{~km} / \mathrm{sec}$ and $2.75 \mathrm{~g} / \mathrm{cm}^{3}$, respectively. The $\kappa_{0}$ for CY14 for $V_{S 30}$ of 760 $\mathrm{m} / \mathrm{sec}$ is $0.039 \mathrm{sec}$. The total FAS site factors for the average hard-rock site condition representative of the KA16 models 1 and 2 are obtained by multiplying the relative hard-rock site factors with the site factors of the CY14 reference site condition and are shown in Figure 6.

\section{Inversion of KA16 Model 1}

The total linear site factors represent the combined effects of the linear site amplification of the $V_{S}$ profile and the attenuation due to damping, parameterized by $\kappa_{0}$. To reduce the trade-off between the $V_{S}$ profile and $\kappa_{0}$ at high frequencies, we assume that the depth dependence of the $V_{S}$ profile follows a power law (e.g., $a \cdot z^{b}$ ). With this assumption, we have use an analytical solution for the combined effects of the site amplification of the $V_{S}$ profile in the top $30 \mathrm{~m}$ and the $\kappa_{0}$ attenuation given the $V_{S 30}$ value. The methodology is described in Al Atik and Abrahamson (2021).

Using the total linear site factors for KA16 model 1 shown in Figure 6, the inversion is performed to estimate the average $\kappa_{0}$ and $V_{S}$ profile representative of the average hard-rock site condition in the model. A zero angle of incidence and a source $V_{S}$ and density of $3.5 \mathrm{~km} / \mathrm{sec}$ and $2.75 \mathrm{~g} / \mathrm{cm}^{3}$, respectively, are used in the inversion. The density- $V_{S}$ relationship used in Al Atik and Abrahamson (2021) is used in this inversion. Because $V_{S 30}$ is unknown for the KA16 models, the inversion is performed to estimate $V_{S 30}$ as well as for different assumed $V_{S 30}$ values. Using the frequency range of 10 to $20 \mathrm{~Hz}$ (10 Hz roughly corresponds to the frequency associated with QWL amplification for the top $30 \mathrm{~m}$ of the profile and $20 \mathrm{~Hz}$ was chosen to avoid the unreliable higher frequencies in the IRVT-based FAS), $\kappa_{0}, V_{S 30}$ and the $V_{S}$ profile in the top $30 \mathrm{~m}$ are estimated analytically by fitting the site response function in the $10-20 \mathrm{~Hz}$ frequency range assuming that the top $30 \mathrm{~m}$ of the $V_{S}$ profile follows a power law function. The estimated $\kappa_{0}$ and $V_{S 30}$ are $0.032 \mathrm{sec}$ and $1300 \mathrm{~m} / \mathrm{sec}$, respectively. Figure 7(a) shows the high-frequency fit compared to the total site factors for frequencies $>10 \mathrm{~Hz}$. For frequencies $<10 \mathrm{~Hz}$, the fit uses the initial site factors as shown in the pink curve.

The site factors modified for frequencies greater than $10 \mathrm{~Hz}$ to follow the highfrequency fit (pink curve in Figure 7(a)) are divided by the $\kappa_{0}$ operator to obtain the linear site amplification function due only to the $V_{S}$ profile which is subsequently smoothed as shown in Figure 7(b). The inverse QWL approach outlined in Al Atik and Abrahamson (2021) is then applied to invert for the $V_{S}$ profile working from high to low frequencies of the site amplification and solving for the shallow to deep layers

Prepared using sagej.cls 
of the profile. The inverted $V_{S}$ profile, which is subsequently smoothed, is shown by the pink curve in Figure 7(c). Because linear site amplifications are considered reliable for frequencies $>0.6 \mathrm{~Hz}$, the $V_{S}$ profile could only be inverted to a depth of $1.06 \mathrm{~km}$. A comparison of the initial relative site factors of KA16 model 1 to those obtained using the inversion results is shown in Figure 7(d). This plot shows that the inverted $V_{S}$ profile and $\kappa_{0}$ representative of the hard-rock condition for KA16 model 1 used along with the reference $V_{S}$ profile and $\kappa_{0}$ for CY14 at $V_{S 30}$ of $760 \mathrm{~m} / \mathrm{sec}$ can approximate reasonably well the initial relative site factors of KA16 model 1 for frequencies up to $30 \mathrm{~Hz}$.

Next, the inversion of KA16 model 1 described in this section is repeated using different assumed $V_{S 30}$ values instead of inverting for $V_{S 30}$ as shown above. This sensitivity analysis allows for a more robust estimation of $\kappa_{0}$ from the high-frequency site factors as well as an evaluation of the range of $V_{S 30}$ and $\kappa_{0}$ values that can fit the hard-rock site factors of KA16 model 1 relative to the reference site condition with $V_{S 30}$ of $760 \mathrm{~m} / \mathrm{sec}$. Assumed $V_{S 30}$ values of 1500, 1700, and $1975 \mathrm{~m} / \mathrm{sec}$ are used in this sensitivity analysis. The value of $1975 \mathrm{~m} / \mathrm{sec}$ is used because it represents the average $V_{S 30}$ of the NGA-East hard-rock data used in KA16. Figure 8 shows a comparison of the initial relative site factors of KA16 model 1 to those obtained using the inversion for the derived and assumed $V_{S 30}$ values. The inversion results for the different assumed $V_{S 30}$ values indicate that, as the assumed $V_{S 30}$ increases, the derived $\kappa_{0}$ value decreases and the slope of the inverted $V_{S}$ profile in the top $30 \mathrm{~m}$ becomes less steep approaching a single constant layer. The sum-of-squared errors (SSE) between the inversion-based relative site factors and the initial site factors in the frequency range of 0.6 to $30 \mathrm{~Hz}$ are calculated and listed in the plots of Figure 8. An evaluation of the SSE values for the different inversion cases as well as the corresponding shapes of the inverted $V_{S}$ profiles indicates that the assumed $V_{S 30}$ of $1975 \mathrm{~m} / \mathrm{sec}$ does not represent the average hard-rock site conditions of KA16 model 1 . The average $V_{S 30}$ of 1975 $\mathrm{m} / \mathrm{sec}$ obtained using the NGA-East hard-rock data in KA16 is likely biased high due to the large number of stations with estimated or assigned $V_{S 30}$ values. As a result, we conclude that, within the context of the QWL approach used in these inversions and the related assumptions made, a $V_{S 30}$ of $1300 \mathrm{~m} / \mathrm{sec}$ (with a range of 1300 to $1500 \mathrm{~m} / \mathrm{sec}$ ) and $\kappa_{0}$ of $0.032 \mathrm{sec}$ (with a range of 0.03 to $0.032 \mathrm{sec}$ ) are representative of the average site conditions of KA16 hard-rock model 1.

Prepared using sagej.cls 


\section{Inversion of KA16 Model 2}

An inversion approach similar to that described in the previous section is applied to estimate the average hard-rock site characteristics representative of KA16 model 2. The first inversion case is performed to estimate $V_{S 30}$ along with $\kappa_{0}$ for the total site factors of KA16 model 2 for the high-frequency range of 12 to $25 \mathrm{~Hz}$ shown in Figure 6 . The frequency range of 12 to $25 \mathrm{~Hz}$ is chosen to capture the smaller- $\kappa_{0}$ scaling expected for this model while staying below the high-frequency limit of $30 \mathrm{~Hz}$. The inversion for KA16 model 2 results in an average $V_{S 30}$ estimate of $1600 \mathrm{~m} / \mathrm{sec}$ and $\kappa_{0}$ of $0.025 \mathrm{sec}$. We note that, for KA16 model 2, the inverted $V_{S 30}$ value is sensitive to the frequency range used to fit the site factors with the analytical function that assumes that the top $30 \mathrm{~m}$ of the $V_{S}$ profile can be approximated with a power-law function. Moreover, the inversion of KA16 model 2 generally required more smoothing than that of model 1 due to the shape of the KA16 model 2 hard-rock factors with bigger jumps in the site factors in the high-frequency range and less smooth transitions.

Next, KA16 model 2 is inverted using different assumed $V_{S 30}$ values of 1500, 1700, 1850,2000 , and $2380 \mathrm{~m} / \mathrm{sec}$. The $V_{S 30}$ of $2380 \mathrm{~m} / \mathrm{sec}$ is reported in KA16 as the average $V_{S 30}$ of the BCHydro data used to derive model 2 scaling factors. Inverted $\kappa_{0}$ values and calculated SSE values for the different inversion cases are listed in Table 1. Similar to the trends observed for model 1, the inversion results for KA16 model 2 indicate that the inverted $\kappa_{0}$ value decreases with increasing $V_{S 30}$ and that KA16 model 2 cannot be well represented with hard-rock conditions with large average $V_{S 30}$ values, particularly greater than $2000 \mathrm{~m} / \mathrm{sec}$. Based on a qualitative evaluation of the inversion results as well as the SSE values for the different cases, we conclude that the inversion results for $V_{S 30}$ of $1700 \mathrm{~m} / \mathrm{sec}$ (range of 1600 to $1850 \mathrm{~m} / \mathrm{sec}$ ) and $\kappa_{0}$ of $0.024 \mathrm{sec}$ (range of 0.022 to $0.025 \mathrm{sec}$ ) best represent the average hard-rock site conditions of KA16 model 2. The best-case inversion results for KA16 model 2 in terms of $\kappa_{0}$ fit, site amplification, and inverted $V_{S}$ profile are shown in Figure 9.

\section{Discussion of Vs Profile and $\kappa_{0}$ Inversions of KA16 Models}

The inverted $V_{S}$ profiles and $\kappa_{0}$ values presented in this section are representative of the average hard-rock site conditions of KA16 models 1 and 2 within the context of the QWL method used in the inversion and the assumptions employed to solve for the multiple unknowns in this process. These assumptions are related to the assigned half-space $V_{S}$ and density values, density- $V_{S}$ relationship, vertical angle of incidence, smooth $V_{S}$ profiles, and the representation of the top $30 \mathrm{~m}$ of the $V_{S}$ profile with a power-law function. While these assumptions are reasonable, they do introduce a level 
of uncertainty in the resulting inverted $V_{S}$ profiles and $\kappa_{0}$ values. Moreover, due the frequency limitations of the KA16 hard-rock factors and their jagged appearance, the inverted $V_{S}$ profiles are limited in their depth range.

Boore (2013) compared site amplifications calculated using the QWL method to those obtained from theoretical simulations of wave propagation in layered media accounting for the constructive and destructive interference of all reverberations in the layers (full resonant [FR] method). For velocity models made up of gradients, Boore (2013) found that the QWL method systematically underestimates the theoretical FR site amplification over a wide frequency range. This underestimation can be on the order of $20 \%$. Based on that, the QWL-based inversion can potentially underestimate the derived $V_{S}$ profiles compared to those expected from the FR method for the same site amplification. The use of the QWL method in the inversion is, however, consistent with the approach used to develop analytical site adjustment factors presented in the next section. Therefore, we consider the inverted profiles and $\kappa_{0}$ values presented in this section as appropriate values for use with the QWL method to represent the average hard-rock site conditions of the KA16 factors. We use these inverted profiles and $\kappa_{0}$ values to constrain the inputs to the analytical calculations for the hard-rock factors.

\section{Development of GMPE-Consistent Analytical Hard-Rock Site Adjustment Factors}

The inversion of the KA16 empirical hard-rock factors indicates that these factors can be used to scale response spectra from a reference $V_{S 30}$ of $760 \mathrm{~m} / \mathrm{sec}$ to target $V_{S 30}$ of about 1300 (model 1) or $1700 \mathrm{~m} / \mathrm{sec}$ (model 2). To develop rock site adjustment factors that are a continuous function of $V_{S 30}$ between 1000 and $2200 \mathrm{~m} / \mathrm{sec}$, we use the analytical IRVT method of Al Atik et al. (2014) with empirical constrains based on the KA16 scaling factors for $V_{S 30}$ of 1300 and $1700 \mathrm{~m} / \mathrm{sec}$ and the NGA-West2 scaling factors for $V_{S 30}$ of $1000 \mathrm{~m} / \mathrm{sec}$. Because the spectral shape for CY14 generally lies in the center of the range of spectral shapes of the NGA-West2 GMPEs, we develop the rock scaling factors using the CY14 GMPE and assume the resulting factors are applicable to the other NGA-West2 GMPEs.

The development of analytical site adjustment factors requires the definition of host and target site conditions in terms of $V_{S}$ profiles and $\kappa_{0}$ values. For the host site condition, the $V_{S}$ profile and $\kappa_{0}$ value of $0.039 \mathrm{sec}$ inverted for CY14 for $V_{S 30}$ of $760 \mathrm{~m} / \mathrm{sec}$ in Al Atik and Abrahamson (2021) are used. Thirteen target site conditions are defined having $V_{S 30}$ ranging between 1000 and $2200 \mathrm{~m} / \mathrm{sec}$. The $V_{S}$ profiles for the target sites are obtained using Boore (2016) based on a $V_{S 30}$-based interpolation

Prepared using sagej.cls 
between generic WUS and Eastern US profiles with $V_{S 30}$ of 618 and $2780 \mathrm{~m} / \mathrm{sec}$, respectively. Figure 10 presents host CY14 $V_{S}$ profile along with the target $V_{S}$ profiles and their corresponding QWL site amplifications. Figure 10 shows that there is a significant difference between the host $V_{S}$ profile for CY14 for $V_{S 30}$ of $760 \mathrm{~m} / \mathrm{sec}$ and the target profile for $V_{S 30}$ of $1000 \mathrm{~m} / \mathrm{sec}$. This difference is due to CY14 having a relatively high $V_{S}$ scaling from 1000 to $760 \mathrm{~m} / \mathrm{sec}$ resulting in higher site amplification and softer $V_{S}$ profile for $V_{S 30}$ of $760 \mathrm{~m} / \mathrm{sec}$ compared to the target profile $V_{S 30}$ of 1000 $\mathrm{m} / \mathrm{sec}$. These effects are discussed in Al Atik and Abrahamson (2021).

\section{Target $\kappa_{0}$}

The estimation of site-specific $\kappa_{0}$ is a complex process that often involves a large degree of uncertainty and trade-offs. The origins of $\kappa_{0}$ and the relationship between the observed high-frequency attenuation in FAS ( $\kappa_{0}$ scaling) and the low-strain damping at a site are subject of ongoing debate. The current paradigm assumes that $\kappa_{0}$, estimated with the source, path, and site effects removed, is due only to damping at the site (EPRI). As a result, a low $\kappa_{0}$ implies low damping that must lead to an increase in the high-frequency ground motion. Hard-rock to soft-rock site factors of 2-3 at the frequency range of $20-40 \mathrm{~Hz}$ are common (Biro and Renault 2012). When the current paradigm was established in the 1990s, there were only a four hard-rock recordings with low $\kappa_{0}$ values and they were consistent with the large factors of 2-3 amplification for hard-rock sites relative to soft-rock sites. The current data sets for hard-rock sites are much larger with over 100 recordings, and they do not show the large site factors at high frequencies that are predicted for hard-rock sites with $\kappa_{0}$ in the 0.006-sec range. This indicates that estimated $\kappa_{0}$ values are not just the result of damping; they also reflect the errors in the assumed source, path, and site effects on the slope of the FAS used to estimate $\kappa_{0}$. The negative values of $\kappa_{0}$ estimated for some sites also indicate that there is more than just damping controlling the $\kappa_{0}$ values (Ktenidou et al. 2021).

To avoid the common tendencies for underestimating $\kappa_{0}$, We use target $\kappa_{0}$ values that are consistent with the observed ground-motion scaling at high-frequencies for rock site conditions. By using the amplitude of the ground motion and not just the high-frequency slope of the FAS, the $\kappa_{0}$ values can be interpreted as effects of damping and used in the traditional $\kappa_{0}$ scaling methodology. We note that our resulting target $\kappa_{0}$ values are not site-specific; they are average values that can be expected for hard-rock sites with different $V_{S 30}$ values. We also account for the uncertainty in the average $\kappa_{0}$ value for a rock site condition as described below.

Prepared using sagej.cls 
For this study, target $\kappa_{0}$ values are estimated based on a review of Silva and Darragh (1995) with additional empirical constrains. Silva and Darragh (1995) analyzed 49 rock sites in WNA and 22 rock sites in Eastern North America [ENA]. Table 5-3 of Silva and Darragh (1995) lists the median and range of $\kappa_{0}$ values for average site conditions in WNA and ENA. It indicates that average $\kappa_{0}$ values for WNA rock site conditions are not small and are larger than those for ENA. Silva and Darragh (1995) interpreted the $\kappa_{0}$ to be the result of damping in the top 1-2 $\mathrm{km}$ below the site and proposed two $\mathrm{Q}$ models $\left(Q=\gamma \cdot V_{S}\right)$ with $\gamma=0.007$ and $0.029 \mathrm{sec} / \mathrm{m}$ for soft-rock and hardrock sites, respectively. Their soft-rock and hard-rock sites are representative WNA and ENA generic $V_{S}$ profiles, respectively, and are shown in Figure 10(a).

For each of the 13 target $V_{S}$ profiles in this study, we estimate $\kappa_{0}$ by summing up the damping in the profile layers over the top 1 and $2 \mathrm{~km}$ of the profile as shown in Equations 1 and 2. Two profile depths are used to capture the uncertainty in the total depth of the profile contributing to damping. Two alternative $\mathrm{Q}$ models are used: a linear Q model with gamma $=0.007 \mathrm{sec} / \mathrm{m}$ representative of WNA soft-rock condition and a bilinear Q model with gamma of $0.007 \mathrm{sec} / \mathrm{m}$ for the profile layers with $V_{S} \leq$ $2700 \mathrm{~m} / \mathrm{sec}$ and $0.029 \mathrm{sec} / \mathrm{m}$ for larger $V_{S}$. This results in a total of four $\kappa_{0}$ estimates for each target $V_{S}$ profile. The alternative target $\kappa_{0}$ estimates as a function of $V_{S 30}$ are shown in Figure 11 (a) and are compared to empirical $\kappa_{0}$ estimates inferred from ground-motion data. Empirical $\kappa_{0}$ estimates shown in Figure 11 are based on $\kappa_{0}$ estimates for the 4 NGA-West2 GMPEs in Al Atik and Abrahamson (2021) for $V_{S 30}$ of 760 and $1000 \mathrm{~m} / \mathrm{sec}$ and on $\kappa_{0}$ and $V_{S 30}$ inverted for the KA16 models. The upper estimates of target $\kappa_{0}$ values for this study shown in Figure 11 are the result of using $\gamma=0.007 \mathrm{sec} / \mathrm{m}$ and a profile depth of $2 \mathrm{~km}$ contributing to damping while the lower estimates are the result of the bilinear Q model with a profile depth of $1 \mathrm{~km}$ contributing to $\kappa_{0}$.

$$
\kappa_{0}=\sum_{i} \frac{H_{i}}{V_{S, i} Q_{i}}
$$

$$
Q=\gamma * V_{S}
$$

Figure 11(a) indicates that the target $\kappa_{0}$ values have a similar trend with $V_{S 30}$ as the empirical $\kappa_{0}$ estimates, but with the average target $\kappa_{0}$ values falling below the average empirical $\kappa_{0}$ estimates, indicating an underestimation of the target $\kappa_{0}$ values compared to the empirical data. Because this study uses CY14 to develop analytical hard-rock site adjustment factors, we constrain the average target $\kappa_{0}$ for $V_{S 30}=1000 \mathrm{~m} / \mathrm{sec}$ to match that of CY14 $(0.0345 \mathrm{sec})$. As a result, the target $\kappa_{0}$ values are scaled up by a

Prepared using sagej.cls 
constant factor and the adjusted target $\kappa_{0}$ values are shown in Figure 11(b). We note that the trend of the empirical $\kappa_{0}$ values as a function of $V_{S 30}$ is still different from that of the scaled target $\kappa_{0}$ values for this study. Our ultimate goal is not to match the exact empirical $\kappa_{0}$ values but to have a good match between the analytical and the empirical rock site adjustment factors. We aim to match the hard-rock scaling observed in empirical data reflecting the combined effects of $\kappa_{0}$ and and $V_{S}$ profile scaling. We also note that the upper estimates of the scaled target $\kappa_{0}$ are within the range of $\kappa_{0}$ values for WNA rock from Silva and Darragh (1995) and are considered reasonable. Table 2 lists the four $\kappa_{0}$ values for the different target $V_{S}$ profiles along with their average and standard deviation.

\section{Hard-Rock Site Adjustment Factors}

For each of the target $V_{S 30}$ values ranging from 1000 to $2200 \mathrm{~m} / \mathrm{sec}$, four sets of adjustment factors are developed using the IRVT approach of Al Atik et al. (2014) corresponding to the four target $\kappa_{0}$ values listed in Table 2. Strike-slip earthquake scenarios with magnitude 5, 6, and 7, distance of 5, 10, and $20 \mathrm{~km}$, and $V_{S 30}$ of 760 $\mathrm{m} / \mathrm{sec}$ are used in the IRVT approach. CY14 median response spectra are computed for the nine scenarios considered for the linear site response. These response spectra are converted into compatible FAS using the IRVT approach as described in the previous sections. Then, each FAS is scaled to adjust for the differences in the linear site amplification and $\kappa_{0}$ scaling between the host and target $V_{S}$ profiles and $\kappa_{0}$ values. The $V_{S}-\kappa$ scaled FAS are then converted into a $V_{S}-\kappa$ scaled response spectra using random vibration theory. The $V_{S}-\kappa$ scaling factors are calculated as the ratio of the scaled response spectra to the initial GMPE response spectra and averaged over the nine scenarios considered.

For each target $V_{S}$ profile, four sets of $V_{S}-\kappa$ scaling factors are computed corresponding to the four target $\kappa_{0}$ values. Average $V_{S^{-}} \kappa$ scaling factors are derived assuming equal weights for the four target $\kappa_{0}$ values. Figure 12 shows the $V_{S}-\kappa$ scaling factors for the individual target $\kappa_{0}$ values as well as the average scaling factors for $V_{S 30}$ of $1700 \mathrm{~m} / \mathrm{sec}$ compared to the empirical hard-rock factors of KA16. Figure 12 indicates a good agreement between the average analytical factors for $V_{S 30}$ of 1700 $\mathrm{m} / \mathrm{sec}$ and the KA16 model 2 factors which have a representative $V_{S 30}$ of about 1700 $\mathrm{m} / \mathrm{sec}$. Figure 13 compares the set of average analytical hard-rock adjustment factors for the range of $V_{S 30}$ of 1000 to $2200 \mathrm{~m} / \mathrm{sec}$ to the CY14 empirical site factors for $V_{S 30}$ of $1000 \mathrm{~m} / \mathrm{sec}$ and the KA16 hard-rock factors. While some mismatch can be observed in Figure 13 between the analytical factors for $V_{S 30}$ of $1300 \mathrm{~m} / \mathrm{sec}$ and the KA16

Prepared using sagej.cls 
model 1 factors, there is good agreement between the analytical hard-rock factors for $V_{S 30}$ of $1000 \mathrm{~m} / \mathrm{sec}$ and the corresponding CY14 site factors for frequencies less than $20 \mathrm{~Hz}$ and between the analytical factors for $V_{S 30}$ of $1700 \mathrm{~m} / \mathrm{sec}$ and the KA16 model 2 factors for $15-30 \mathrm{~Hz}$. We conclude that, on average, the analytical hard-rock factors are reasonable based on their comparison with empirical scaling for rock site conditions (CY14 for $V_{S 30}=1000 \mathrm{~m} / \mathrm{sec}$ and KA16 factors).

\section{Implementation}

The hard-rock site adjustment factors derived in this study are used to extrapolate the average NGA-West 2 empirical site factors to hard-rock conditions in a relative sense to ensure a smooth transition in the scaling factors to hard-rock sites. As such, the ratios of hard-rock analytical factors relative to those for $V_{S 30}$ of $1000 \mathrm{~m} / \mathrm{sec}$ are used to model the scaling of the hard-rock site factors. These ratios are then applied to the empirical site factors for $V_{S 30}$ of $1000 \mathrm{~m} / \mathrm{sec}$ relative to reference $V_{S 30}=760 \mathrm{~m} / \mathrm{sec}$. This normalization of the analytical site factors allows the site factors from the analytical modeling to be centered on the GMPEs which provides a smooth scaling from soft-rock to hard-rock site conditions. The empirical linear site factors for $V_{S 30}$ of $1000 \mathrm{~m} / \mathrm{sec}$ are obtained by averaging the ratio of median response spectra for $V_{S 30}$ of $1000 \mathrm{~m} / \mathrm{sec}$ relative to $760 \mathrm{~m} / \mathrm{sec}$ for 4 NGA-West2 GMPEs (Abrahamson et al. 2014; Boore et al. 2014; Campbell and Bozorgnia 2014; Chiou and Youngs 2014). The resulting rock-site adjustment factors are shown in Figure 14 and included as an electronic appendix to this paper. Figure 14 also shows the average empirical linear site factors of the NGA-West2 GMPEs for $V_{S 30}$ of 680 to $1000 \mathrm{~m} / \mathrm{sec}$ relative to the reference $760 \mathrm{~m} / \mathrm{sec}$. The GMPEs nonlinear site response is not included in the calculation of the average empirical site factors. Figure 14 indicates a smooth extrapolation of the empirical average GMPE site factors to hard-rock conditions based on the analytical factors described in this paper.

Figure 15 shows the linear $V_{S}$ scaling of the NGA-West2 GMPEs relative to $V_{S 30}$ of $760 \mathrm{~m} / \mathrm{sec}$ and extrapolated to hard-rock conditions. Also plotted in Figure 15 are the average of the scaling from the 4 NGA-West2 GMPEs and the hard-rock scaling proposed in this study. Comparisons of the linear $V_{S}$ scaling are shown for frequencies of $0.2,1,5$, and $25 \mathrm{~Hz}$. These comparisons indicate that, for $V_{S 30}$ values $>1000$ $\mathrm{m} / \mathrm{sec}$, linear $V_{S}$ scaling varies among the NGA-West2 GMPEs reflecting the different hard-rock extrapolation constraints imposed in the models. The extrapolated hard-rock scaling in the NGA-West2 GMPEs is unconstrained with empirical data for hard-rock conditions and is, therefore, unreliable for application to hard-rock sites. In contrast to the hard-rock factors proposed in this study, the NGA-West2 scaling does not follow 
expected trends with $\kappa$ for hard-rock sites at the high frequency of $25 \mathrm{~Hz}$ as shown in Figure 15 (a). Therefore, the NGA-West2 linear $V_{S}$ scaling should not be extrapolated to hard-rock sites and the factors presented in this paper should be used instead.

The average hard-rock adjustment factors from this study, presented in Figure 14 and included as an electronic appendix to this paper, can be applied to correct the average median ground motion predicted by the NGA-West 2 GMPEs with $V_{S 30}$ of $760 \mathrm{~m} / \mathrm{sec}$ to a hard-rock site with $V_{S 30}$ between 1000 and $2200 \mathrm{~m} / \mathrm{sec}$. Nonlinear site response should be disabled when calculating the NGA-West2 ground-motion predictions for $V_{S 30}$ of $760 \mathrm{~m} / \mathrm{sec}$ before applying the hard-rock adjustment factors. For target $V_{S 30}$ values not explicitly listed in the electronic appendix, hard-rock factors can be obtained using a log-log interpolation of the provided factors for the neighboring $V_{S 30}$ values. For hard-rock sites with qualitative assessment of site conditions, hard-rock adjustment factors for a range of target $V_{S 30}$ values can be enveloped to estimate the median hardrock adjustment factors.

\section{Site-to-Site Uncertainty}

The adjustment of median ground-motion predictions for hard-rock sites is presented in this paper. To evaluate the uncertainty in the hard-rock adjustment factors, we examine the site-to-site variability $\left[\phi_{S 2 S}\right]$ in the NGA-West2 GMPEs for soil versus rock sites. Site terms are obtained using a mixed-effects regression on the within-event residuals of the NGA-West 2 GMPEs with the station term as the random effect and using earthquakes with magnitude $>=5$ and stations with a minimum of 3 recordings as described in Al Atik (2015). Ground-motion data with magnitude $<3$ are not used in this analysis to reduce the dependence of linear site factors on earthquake magnitude. This effect was examined in Stafford et al. (2017) and was found to be most pronounced at short periods and for small magnitude scenarios. Soil sites in the NGA-West 2 database are classified with $V_{S 30}<680 \mathrm{~m} / \mathrm{sec}$ while rock sites have $V_{S 30}$ $\geq 680 \mathrm{~m} / \mathrm{sec}$. Site terms for each NGA-West 2 GMPE are divided in these two site categories and the resulting $\phi_{S 2 S}$ are computed.

The $\phi_{S 2 S}$ for soil and rock sites obtained using the residuals of ASK14, Boore et al. (2014) [BSSA14] and CY14 for magnitude $\geq 5$ were examined and the comparison using CY14 residuals is shown in Figure 16. We note $\phi_{S 2 S}$ for Campbell and Bozorgnia (2014) [CB14] is not shown due to the limited CB14 dataset as a result of restricting the residuals to magnitudes $>5$ and stations with a minimum of 3 recordings. This impacted the stability of the $\phi_{S 2 S}$ estimates for CB14. The large error bars in Figure 16 reflect the smaller subset of stations with $V_{S 30} \geq 680 \mathrm{~m} / \mathrm{sec}$ compared to the number

Prepared using sagej.cls 
of softer sites in the NGA-West2 dataset. For example, using the CY14 residuals, the average $V_{S 30}$ is about $390 \mathrm{~m} / \mathrm{sec}$ for soil sites and $830 \mathrm{~m} / \mathrm{sec}$ for rock sites. The comparison of $\phi_{S 2 S}$ for soil and rock sites indicates that the NGA-West $2 \phi_{S 2 S}$ values are generally comparable for the two site groups at high frequencies as shown in Figure 16. At periods greater than $1 \mathrm{sec}, \phi_{S 2 S}$ values for rock sites are lower than those for soil sites. We note that the subsets of data for rock sites are very limited in number of stations for periods $>4 \mathrm{sec}$. We conclude that, for hazard significant scenarios with magnitudes $\geq 5, \phi_{S 2 S}$ obtained from the NGA-West 2 residuals for all $V_{S 30}$ can be used to estimate $\phi_{S 2 S}$ for hard-rock sites with modifications associated with the expected spectral shapes of site variability for hard-rock.

The average $\phi_{S 2 S}$ obtained using residuals of ASK14, BSSA14 and CY14 for magnitude $\geq 5$ and for all $V_{S 30}$ values is shown in Figure 17 (a). We note that the peak in $\phi_{S 2 S}$ at frequency $5-10 \mathrm{~Hz}$ is likely related to the variability of the resonance frequency of shallow layers for soil and soft-rock sites. For hard-rock sites, this peak is expected to be shifted to higher frequencies reflecting the variability in kappa scaling for hard-rock conditions. We examine this effect using $\phi_{S 2 S}$ obtained from a residual analysis of Japanese surface and borehole data. A discussion of the residual analysis of the Japanese dataset is presented in Goulet et al. (2018). Figure 17 (b) presents a comparison of $\phi_{S 2 S}$ for the surface and borehole Japanese data with magnitude $>=5$. Borehole $\phi_{S 2 S}$ values obtained using stations with $V_{S}>=1000 \mathrm{~m} / \mathrm{sec}$ are also shown. Figure 17 (b) shows a shift in the peak of $\phi_{S 2 S}$ to higher frequencies for the rock borehole data compared to the surface data. As a result, we correct the average $\phi_{S 2 S}$ for NGA-West 2 to follow the high-frequency scaling of the Japanese borehole $\phi_{S 2 S}$ for frequencies greater than $2.5 \mathrm{~Hz}$. For frequencies less than $2.5 \mathrm{~Hz}$, the $\phi_{S 2 S}$ shape is based on the NGA-West 2 data. The resulting proposed $\phi_{S 2 S}$ model for use for hardrock sites is shown in Figure 17 (a) and listed in Table 3. This proposed model can be used to characterize the epistemic uncertainty of the average rock-site adjustment factors presented in this study if additional site-specific information is not available to constrain the epistemic uncertainty of the site factors.

For hard-rock adjustments of the NGA-West2 GMPEs using the ergodic aleatory variability model, the standard deviation models in the NGA-West2 GMPEs, calculated for $V_{S 30}$ of $760 \mathrm{~m} / \mathrm{sec}$ without including effects of nonlinear site response, could be used for hard-rock sites. The use of the ergodic NGA-West 2 sigma models is likely conservative for some frequency ranges and might not capture the expected peaks in the variability for hard-rock sites. Alternatively, ergodic sigma for hard-rock sites can be constructed using $\phi_{S 2 S}$ proposed in this study along with NGA-West 2 Tau models and published single-station sigma models for WUS ((Al Atik 2015)). We note that the

Prepared using sagej.cls 
$\phi_{S 2 S}$ model proposed in this study for hard-rock site adjustment factors is a simplified model based on adjusting the NGA-West $2 \phi_{S 2 S}$. A more detailed study of the groundmotion variability and its components for hard-rock sites is warranted.

\section{Conclusions and Discussion}

Hard-rock adjustment factors are derived to adjust the NGA-West2 GMPEs from their average host site conditions with $V_{S 30}$ of $760 \mathrm{~m} / \mathrm{sec}$ to target sites with $V_{S 30}$ ranging from 1000 to $2200 \mathrm{~m} / \mathrm{sec}$. These analytical factors are obtained using the IRVT approach Al Atik et al. (2014) and are consistent with empirical scaling observed in ground-motion data. These factors can be applied to adjust median NGA-West2 ground motions at $V_{S 30}$ of $760 \mathrm{~m} / \mathrm{sec}$ to hard-rock conditions and can be assumed to have the same overall site-to-site uncertainty inherent in the NGA-West2 GMPEs.

The site adjustment factors developed in this study are computed using generic $V_{S}$ profiles and $\kappa_{0}$ values that would be representative of average site response in WUS for rock site conditions. The KA16 Scaling factors obtained using ENA and BCHydro data are used as empirical constraints for this study because of the scarcity of empirical data on hard-rock sites in WUS and because KA16 showed that average hard-rock scaling in ENA is comparable to what would be expected for WUS sites. The proposed hard-rock factors are intended for use at sites with measured or estimated $V_{S 30}$ or sites with qualitative assessment of site condition. For hard-rock sites with site-specific measurements of $V_{S}$ profiles extending below the shallow 20 to $30 \mathrm{~m}$ of the profile, the hard-rock adjustment factors presented here are not recommended to be used. For such sites, site-specific adjustments need to be developed following a characterization of the target site-specific conditions in terms of best estimates and uncertainty of $V_{S}$ profiles and $\kappa_{0}$. Also, the use of $\phi_{S 2 S}$ for site-specific adjustments is conservative and can potentially be reduced based on the uncertainty in the site-specific characterization.

\section{Data and Resources}

The pyrvt program used to perform the inverse random vibration theory (IRVT) and random vibration theory (RVT) calculations (Kottke 2020). An Excel file containing the hard rock adjustment factors for the NGA-West2 GMPEs is included as a supplemental material.

Prepared using sagej.cls 


\section{Acknowledgments}

This study was sponsored by Pacific Gas \& Electric (PG\&E). The authors gratefully acknowledge this funding. Any opinions, findings, and conclusions or recommendations expressed in this material are those of the authors and do not necessarily reflect those of the sponsoring company.

\section{References}

Abrahamson NA, Silva WJ and Kamai R (2014) Summary of the ask14 ground-motion relation for active crustal regions. Earthquake Spectra 30(3): 1025-1055.

Al Atik L (2015) NGA-East: Ground-motion standard deviation models for central and eastern north america. PEER Report 2015(7).

Al Atik L and Abrahamson N (2021) A methodology for the development of 1D reference $v_{S}$ profiles compatible with ground-motion prediction equations: Application to NGA-West2 GMPEs. Bulletin of the Seismological Society of America 111(4): 1765-1783.

Al Atik L, Kottke A, Abrahamson N and Hollenback J (2014) Kappa ( $\kappa$ ) scaling of groundmotion prediction equations using an inverse random vibration theory approach. Bulletin of the Seismological Society of America 104(1): 336-346.

Biro Y and Renault P (2012) Importance and impact of host-to-target conversions for ground motion prediction equations in PSHA. Proceedings of the 15th World Conference on Earthquake Engineering, Lisboa 2012 : 24-28.

Boore DM (2003) Simulation of ground motion using the stochastic method. Pure Applied Geophysics 160: 635-676.

Boore DM (2013) The uses and limitations of the square-root impedance method for computing site amplification. Bulletin of the Seismological Society of America 103(4): 2356-2368.

Boore DM (2016) Determining generic velocity and density models for crustal amplification calculations, with an update of the boore and joyner (1997) generic site amplification for $\operatorname{vs}(z)=760$ m/s. Bulletin of the Seismological Society of America 106(1): 316-320.

Boore DM, Stewart JP, Seyhan E and Atkinson GM (2014) Nga-west2 equations for predicting pga, pgv, and 5\% damped psa for shallow crustal earthquakes. Earthquake Spectra 30(3): $1057-1085$.

Campbell KW (2003) Prediction of strong ground motion using the hybrid empirical method and its use in the development of ground motion (attenuation) relations in eastern north america. Bulletin of the Seismological Society of America 93(3): 1012-1033.

Campbell KW and Bozorgnia Y (2014) Nga-west2 ground motion model for the average horizontal components of pga, pgv, and 5\% damped linear acceleration response spectra.

Prepared using sagej.cls 
Earthquake Spectra 30(3): 1087-1115.

Chiou BSJ and Youngs RR (2014) Update of the chiou and youngs NGA model for the average horizontal component of peak ground motion and response spectra. Earthquake Spectra 30(3): 1117-1153.

(EPRI) EPRI (1993) Guidelines for determining design basis ground motions. Report TR102293, Electric Power Research Institute (EPRI), Palo Alto, CA.

Goulet C, Bozorgnia Y, Abrahamson N, Kuehn N, Al Atik L, Youngs R and Graves R (2018) Central and eastern north america ground-motion characterization - NGA-East final report. PEER Report 2018(8).

Kottke A (2020) arkottke/pyrvt v0.7.2. DOI:10.5281/zenodo.3630729. URL https: / / doi . org/10.5281/zenodo.3630729.

Ktenidou OJ and Abrahamson N (2016) Empirical estimation of high-frequency ground motion on hard rock. Seismological Research Letters 87(6): 1465-1478.

Ktenidou OJ, Abrahamson NA, J SW, Darragh RB and Kishida T (2021) The search for hardrock kappa $(\kappa)$ in NGA-East: A semi-automated method for large, challenging datasets in stable continental regions. Earthquake Spectra 37(1): 1391-1419.

Silva WJ and Darragh R (1995) Engineering characterization of strong ground motion recorded at rock sites. Pacific Engineering and Analysis, Inc., El Cerrito, CA (United States) .

Stafford PJ, Rodriguez-Marek A, Edwards B, Rodriguez-Marek A, Kruiver P and Bommer J (2017) Scenario dependence of linear site effect factors for short-period response spectral ordinates. Bulletin of the Seismological Society of America 107(6): 2859-2872.

Vanmarke EH (1975) On the distribution of the first-passage time for normal stationary random processes. Journal of Applied Geophysics 42(1): 215-220.

Prepared using sagej.cls 
Table 1. Results of the inversion for KA16 model 2 for the different cases analyzed.

\begin{tabular}{|c|c|c|c|}
\hline Case & $V_{S 30}(\mathrm{~m} / \mathrm{sec})$ & Inverted $\kappa_{0}(\mathrm{sec})$ & SSE $(0.6$ to $30 \mathrm{~Hz})$ \\
\hline Inverted $V_{S 30}$ & 1602 & 0.025 & 0.325 \\
\hline Assumed $V_{S 30}$ & 1500 & 0.026 & 0.371 \\
\hline Assumed $V_{S 30}$ & 1700 & 0.024 & 0.350 \\
\hline Assumed $V_{S 30}$ & 1850 & 0.022 & 0.414 \\
\hline Assumed $V_{S 30}$ & 2000 & 0.021 & 0.477 \\
\hline Assumed $V_{S 30}$ & 2380 & 0.019 & 0.769 \\
\hline
\end{tabular}

Table 2. Target $\kappa_{0}$ values used in the development of the analytical rock site adjustment factors.

\begin{tabular}{|c|c|c|c|c|c|c|}
\hline $\begin{array}{c}V_{S 30} \\
(\mathrm{~m} / \mathrm{sec})\end{array}$ & $\kappa_{0}-1(\mathrm{sec})$ & $\kappa_{0}-2(\mathrm{sec})$ & $\kappa_{0}-3(\mathrm{sec})$ & $\kappa_{0}-4(\mathrm{sec})$ & $\begin{array}{c}\text { Average } \kappa_{0} \\
(\mathrm{sec})\end{array}$ & $\begin{array}{c}\text { Standard } \\
\text { Deviation } \\
(\mathrm{LN} \text { units })\end{array}$ \\
\hline 1100 & 0.0296 & 0.0462 & 0.0235 & 0.0276 & 0.0307 & 0.289 \\
\hline 1200 & 0.0275 & 0.0436 & 0.0206 & 0.0245 & 0.0279 & 0.322 \\
\hline 1300 & 0.0258 & 0.0416 & 0.0182 & 0.0221 & 0.0256 & 0.353 \\
\hline 1400 & 0.0245 & 0.0399 & 0.0162 & 0.0200 & 0.0237 & 0.387 \\
\hline 1500 & 0.0233 & 0.0385 & 0.0144 & 0.0182 & 0.0220 & 0.420 \\
\hline 1600 & 0.0223 & 0.0373 & 0.0129 & 0.0165 & 0.0205 & 0.458 \\
\hline 1700 & 0.0215 & 0.0363 & 0.0116 & 0.0153 & 0.0193 & 0.490 \\
\hline 1800 & 0.0208 & 0.0354 & 0.0106 & 0.0142 & 0.0182 & 0.521 \\
\hline 1900 & 0.0202 & 0.0346 & 0.0095 & 0.0131 & 0.0172 & 0.560 \\
\hline 2000 & 0.0196 & 0.0339 & 0.0088 & 0.0124 & 0.0164 & 0.584 \\
\hline 2100 & 0.0192 & 0.0333 & 0.0082 & 0.0117 & 0.0157 & 0.611 \\
\hline 2200 & 0.0187 & 0.0327 & 0.0075 & 0.0110 & 0.0150 & 0.640 \\
\hline
\end{tabular}


Table 3. Proposed site-to-site uncertainty model $\left(\phi_{S 2 S}\right)$ model.

\begin{tabular}{|c|c|c|}
\hline Frequency $(\mathrm{Hz})$ & Period (sec) & $\phi_{S 2 S}$ (LN units) \\
\hline 100.00 & 0.010 & 0.3110 \\
\hline 50.00 & 0.020 & 0.3110 \\
\hline 33.33 & 0.030 & 0.3275 \\
\hline 20.00 & 0.050 & 0.3901 \\
\hline 13.33 & 0.075 & 0.3894 \\
\hline 10.00 & 0.100 & 0.3627 \\
\hline 6.67 & 0.150 & 0.3308 \\
\hline 5.00 & 0.200 & 0.3182 \\
\hline 4.00 & 0.250 & 0.3182 \\
\hline 3.33 & 0.300 & 0.3182 \\
\hline 2.50 & 0.400 & 0.3182 \\
\hline 2.00 & 0.500 & 0.3312 \\
\hline 1.33 & 0.750 & 0.3446 \\
\hline 1.00 & 1.000 & 0.3739 \\
\hline 0.67 & 1.500 & 0.4001 \\
\hline 0.50 & 2.000 & 0.4185 \\
\hline 0.33 & 3.000 & 0.4232 \\
\hline 0.25 & 4.000 & 0.4065 \\
\hline 0.20 & 5.000 & 0.3965 \\
\hline 0.13 & 7.500 & 0.3480 \\
\hline 0.10 & 10.000 & 0.2877 \\
\hline & & \\
\hline & & \\
\hline & & 0.000 \\
\hline
\end{tabular}




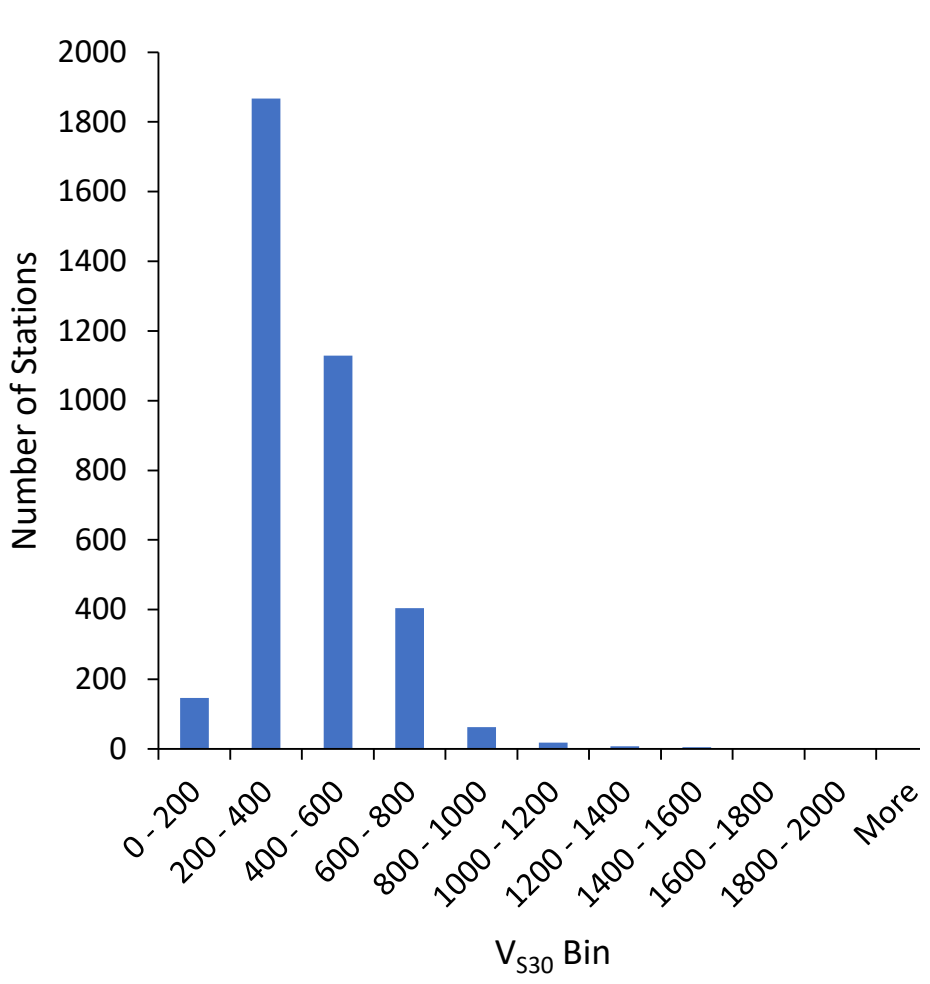

Figure 1. Histogram of the number of stations in different $V_{S 30}$ bins in the ASK14 dataset.

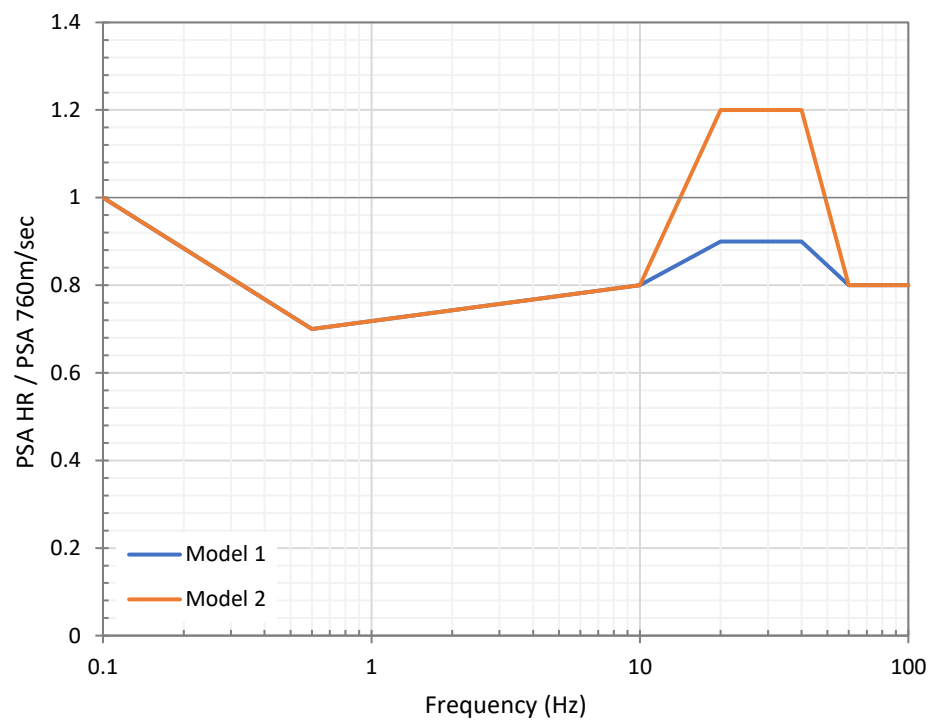

Figure 2. KA16 hard-rock scaling factors relative to $V_{S 30}$ of $760 \mathrm{~m} / \mathrm{sec}$. 


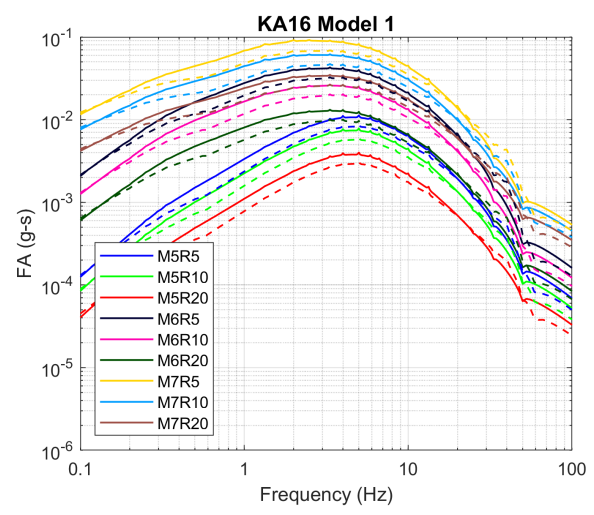

(a)

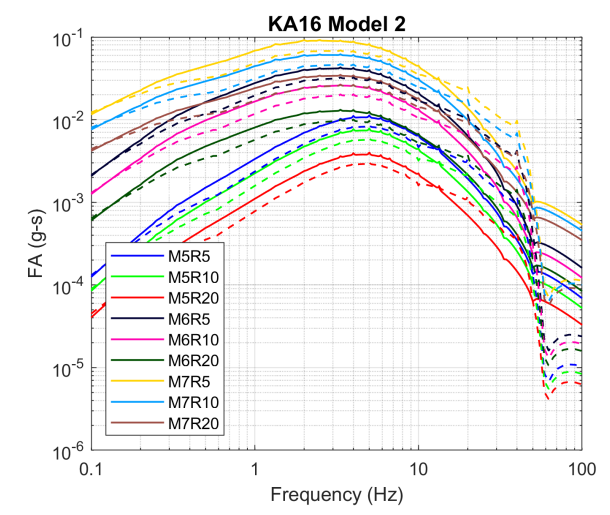

(b)

Figure 3. CY14 IRVT-based Fourier amplitude spectra for $V_{S 30}=760 \mathrm{~m} / \mathrm{sec}$ (solid lines) and for spectra corrected to hard-rock conditions (dashed lines) using KA16 model 1 (a) and model 2 (b).

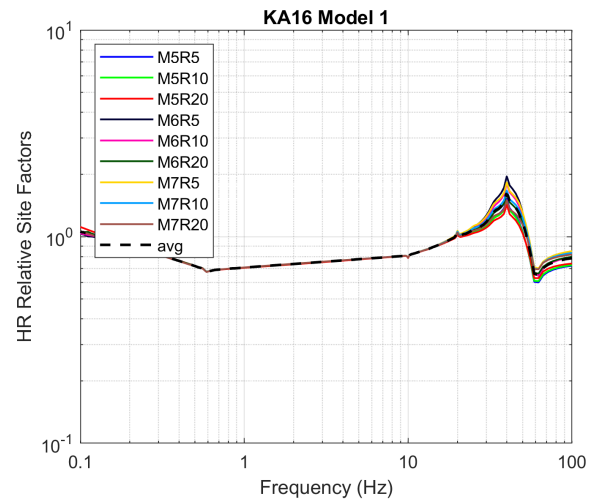

(a)

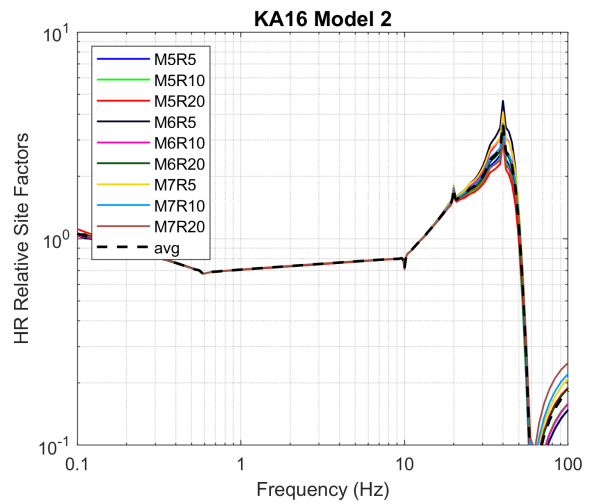

(b)

Figure 4. Hard-rock site factors in FAS domain relative to $V_{S 30}=760 \mathrm{~m} / \mathrm{sec}$ for a suite of scenarios (solid lines) and average relative site factors over all scenarios (dashed lines) for KA16 model 1 (a) and model 2 (b). 


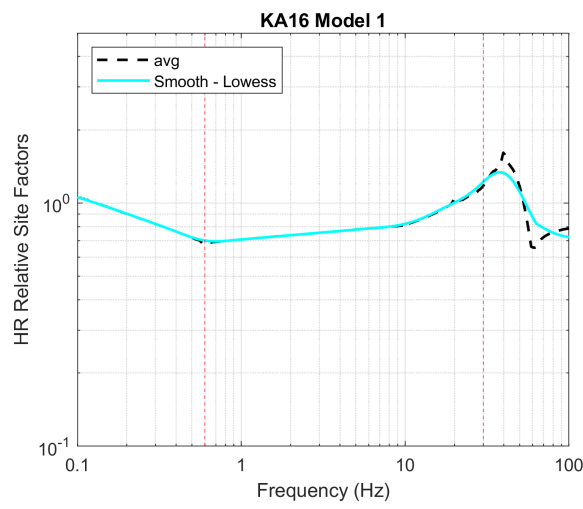

(a)

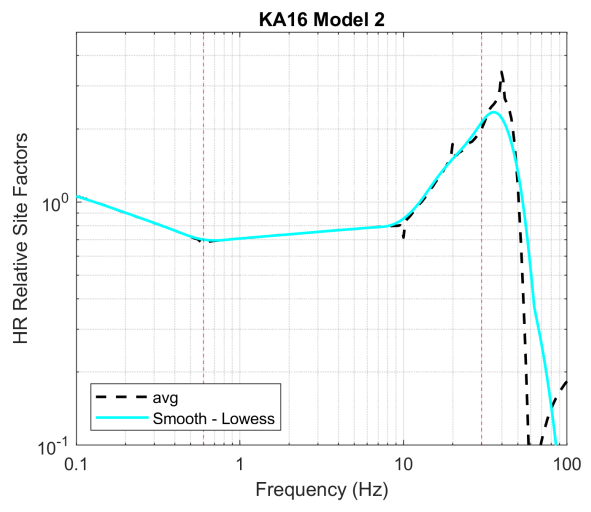

(b)

Figure 5. Average hard-rock site factors relative to $760 \mathrm{~m} / \mathrm{sec}$ in FAS domain (dashed lines) and smoothed factors (solid lines) for KA16 model 1 (a) and model 2 (b). Dashed red vertical lines indicate the frequency range used in the analysis $(0.6$ to $30 \mathrm{~Hz})$.

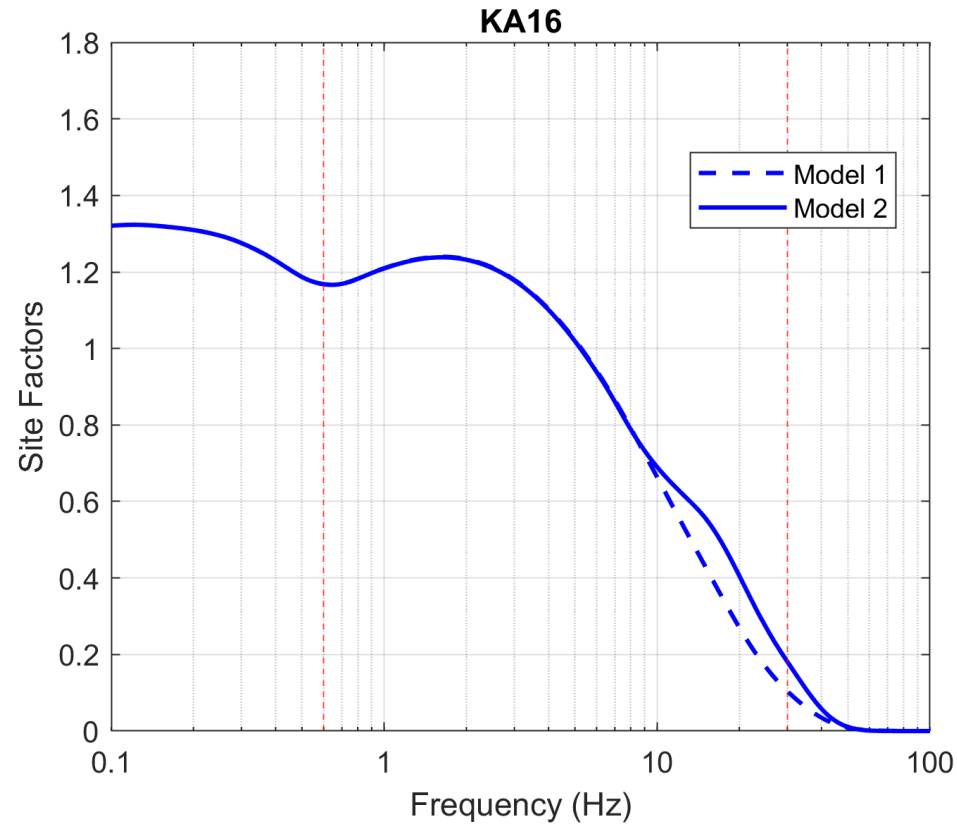

Figure 6. Total FAS site factors for the average hard-rock site conditions representative of the KA16 models. Dashed red vertical lines indicate the reliable frequency range (0.6 to 30 $\mathrm{Hz})$. 

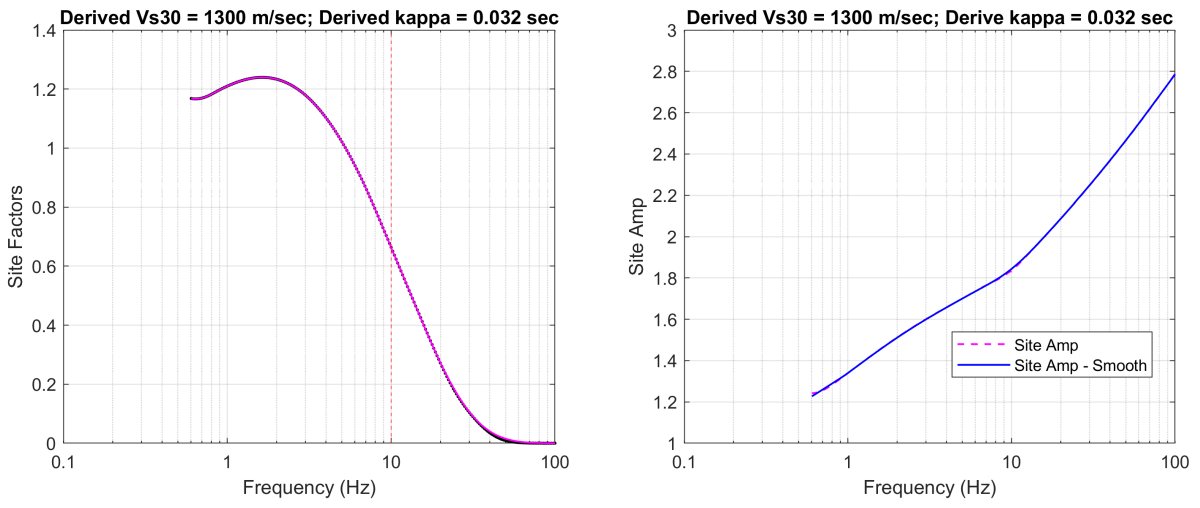

(a)

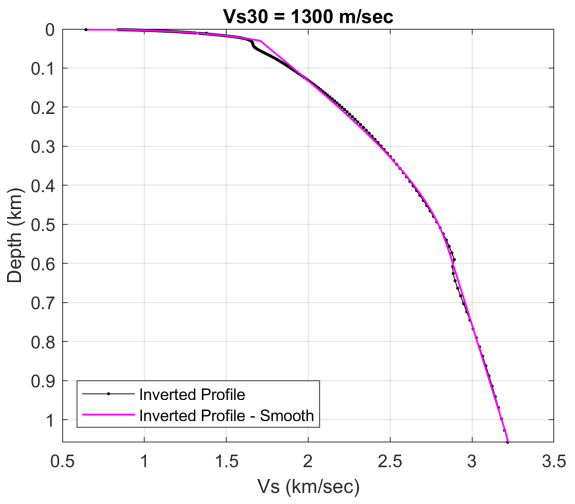

(b)

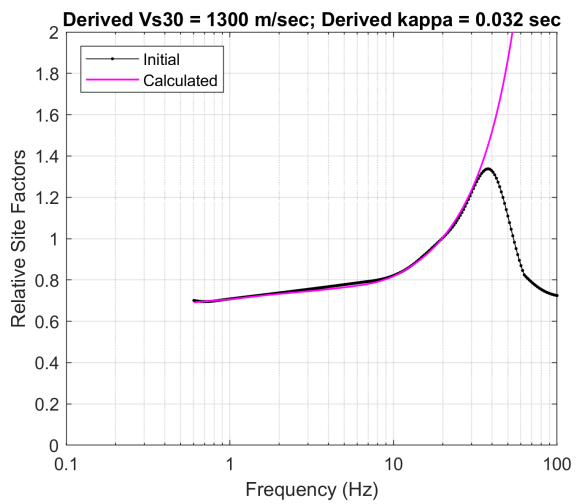

(c)

(d)

Figure 7. Inversion results for KA16 model 1. (a) Hard-rock site factors and high-frequency fit to estimate $\kappa_{0}$ and $V_{S 30}$. (b) Site amplification function obtained by dividing the fitted site factors by the $\kappa_{0}$ operator. (c) Inverted $V_{S}$ profile and smoothed. (d) comparison of the hard-rock site factors relative to $V_{S 30}$ of $760 \mathrm{~m} / \mathrm{sec}$ obtained from the inversion (calculated) to the initial relative site factors. 


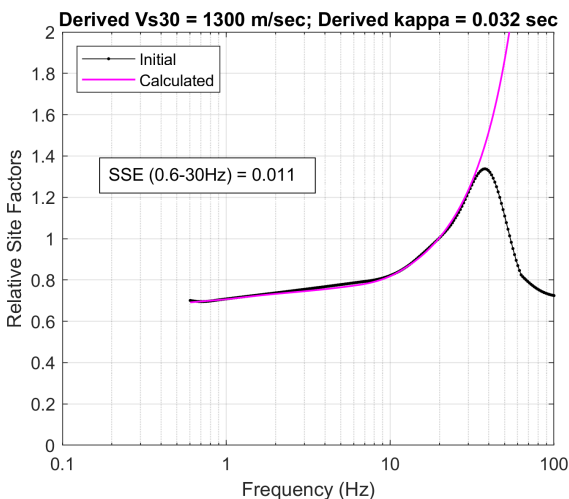

(a)

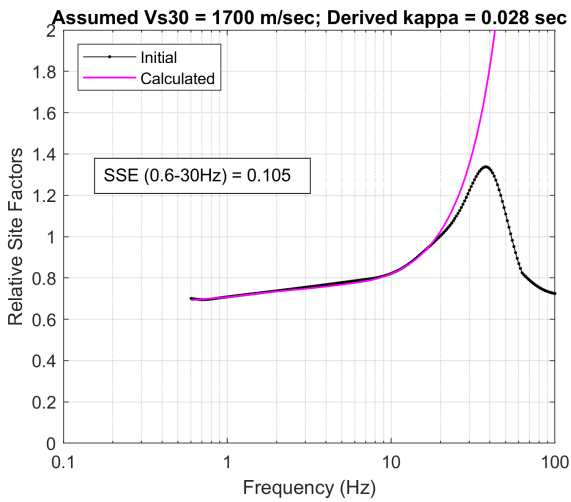

(c)

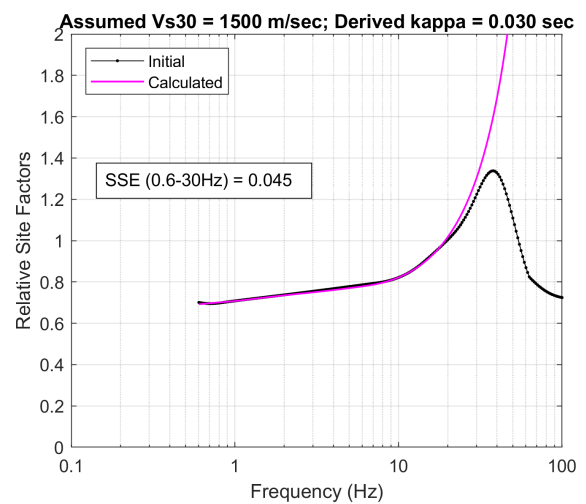

(b)

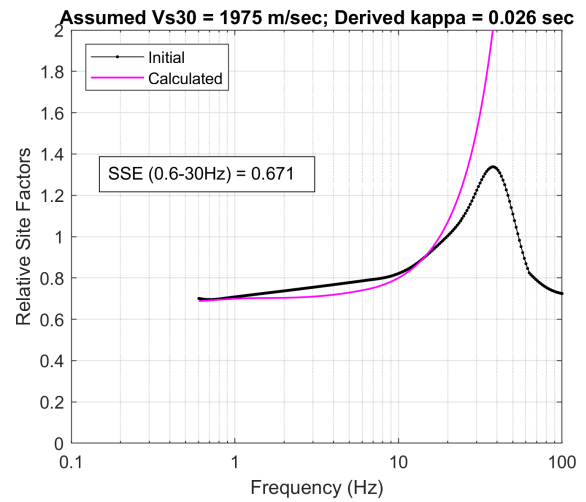

(d)

Figure 8. Comparison of the KA16 model 1 hard-rock site factors relative to $V_{S 30}$ of 760 $\mathrm{m} / \mathrm{sec}$ to the relative site factors obtained from the inversions for the cases of (a) derived $V_{S 30}$ and assumed $V_{S 30}$ values of (b) 1500 , (c) 1700 , and (d) $1975 \mathrm{~m} / \mathrm{sec}$. Derived $\kappa_{0}$ values and calculated SSE are included in the plots. 

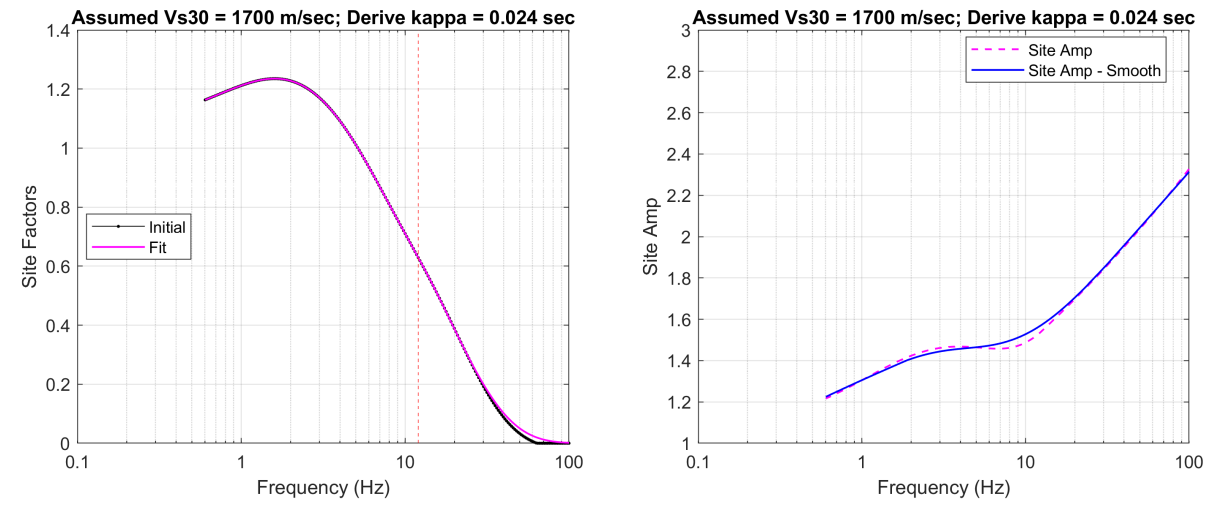

(a)

(b)
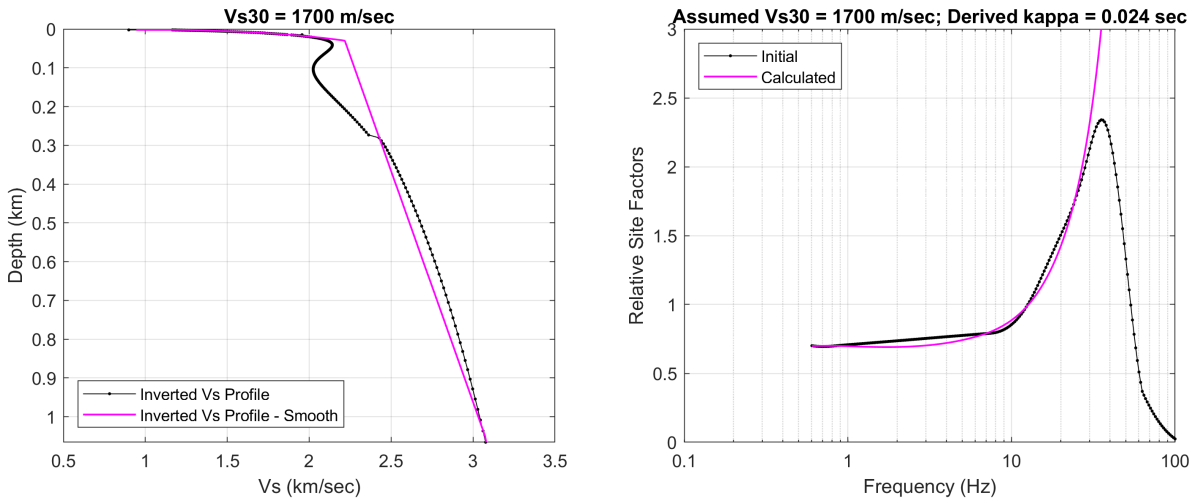

(c)

(d)

Figure 9. Inversion results for KA16 model 2. (a) Hard-rock site factors and high-frequency fit to estimate $\kappa_{0}$ for an assumed $V_{S 30}$ of $1700 \mathrm{~m} / \mathrm{sec}$. (b) Site amplification function obtained by dividing the fitted site factors by the $\kappa_{0}$ operator. (c) Inverted $V_{S}$ profile and smoothed. (d) comparison of the hard-rock site factors relative to $V_{S 30}$ of $760 \mathrm{~m} / \mathrm{sec}$ obtained from the inversion (calculated) to the initial relative site factors. 


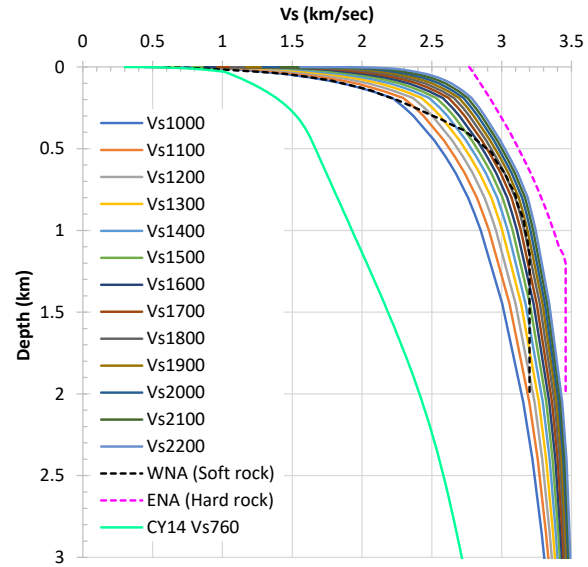

(a)

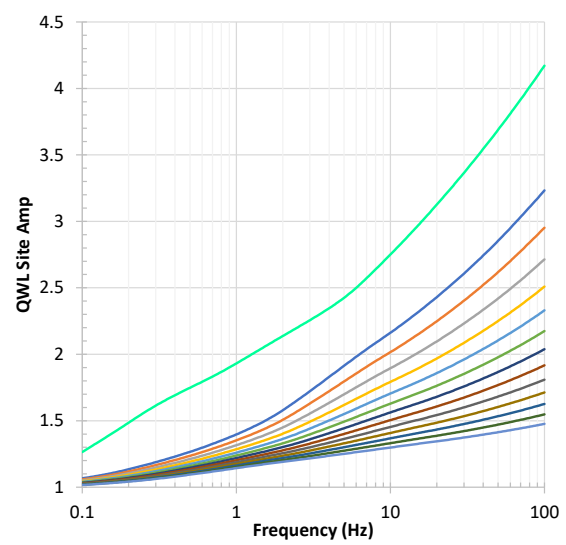

(b)

Figure 10. (a) Host (CY14 Vs760) and target $V_{S}$ profiles compared to the WNA and ENA $V_{S}$ profiles of Silva and Darragh (1995) (b) Corresponding QWL linear site amplification.

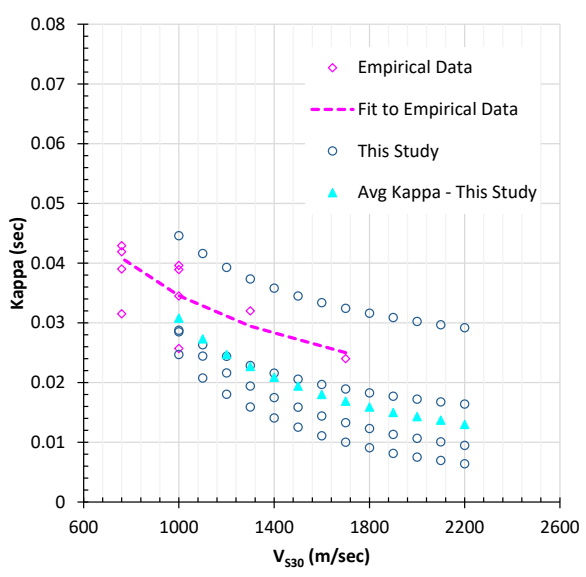

(a)

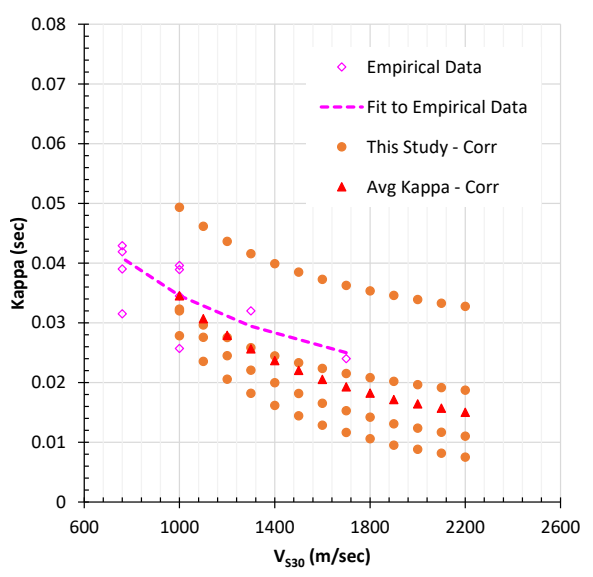

(b)

Figure 11. (a) Comparison of target $\kappa_{0}$ values as a function of $V_{S 30}$ to $\kappa_{0}$ inferred from empirical ground motion data (b) Scaled target $\kappa_{0}$ values such that their average matches CY14 $\kappa_{0}$ at $V_{S 30}$ of $1000 \mathrm{~m} / \mathrm{sec}$. 


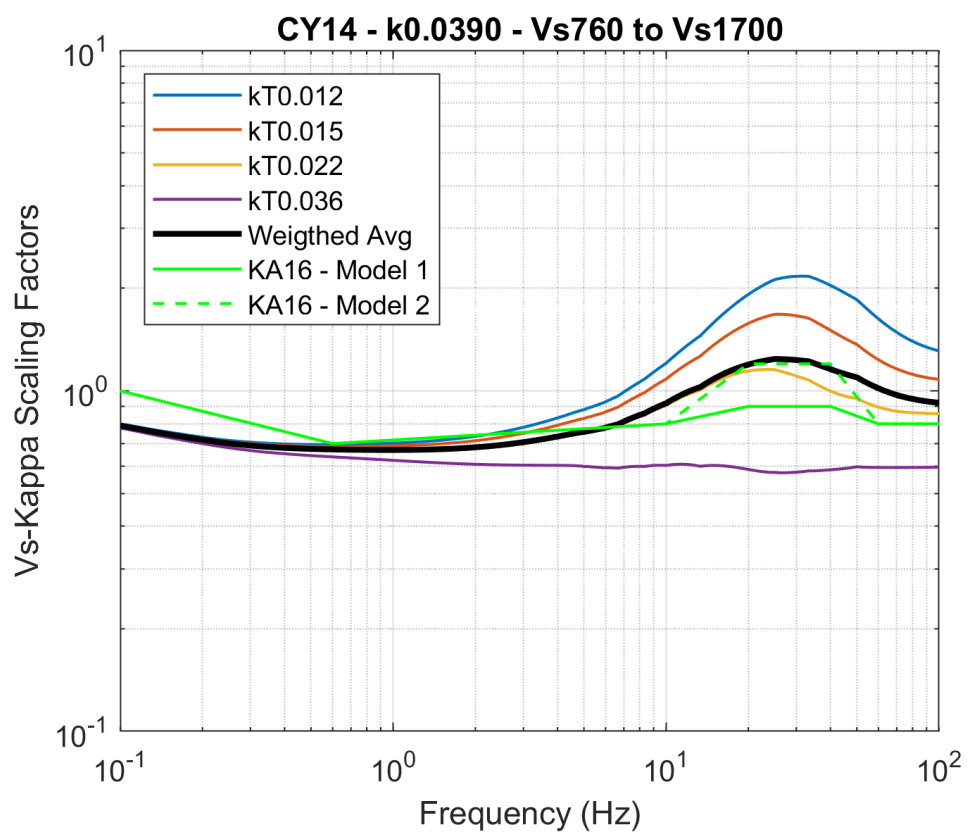

Figure 12. Comparison of the analytical adjustment factors for target $V_{S 30}=1700 \mathrm{~m} / \mathrm{sec}$ to the KA16 rock site adjustment factors.

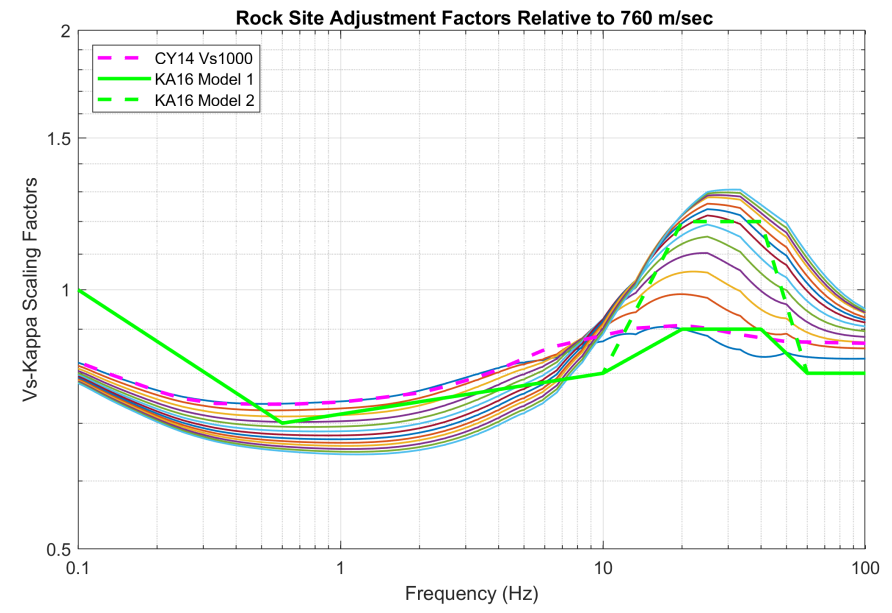

Figure 13. Analytical hard-rock site adjustment factors for target $V_{S 30}$ of 1000 to 2200 $\mathrm{m} / \mathrm{sec}$ compared to the CY14 site factors for $V_{S 30}=1000 \mathrm{~m} / \mathrm{sec}$ and the KA16 hard-rock rock site adjustment factors. 


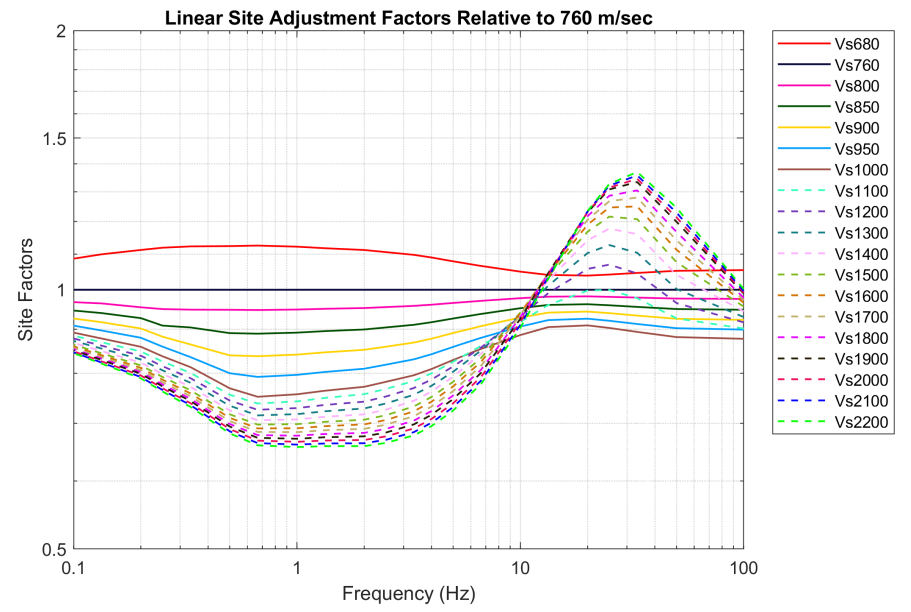

Figure 14. Proposed linear site adjustment factors for $V_{S 30}=680$ to $2200 \mathrm{~m} / \mathrm{sec}$ relative to $760 \mathrm{~m} / \mathrm{sec}$. Solid and dashed lines show empirical and analytical factors, respectively. 

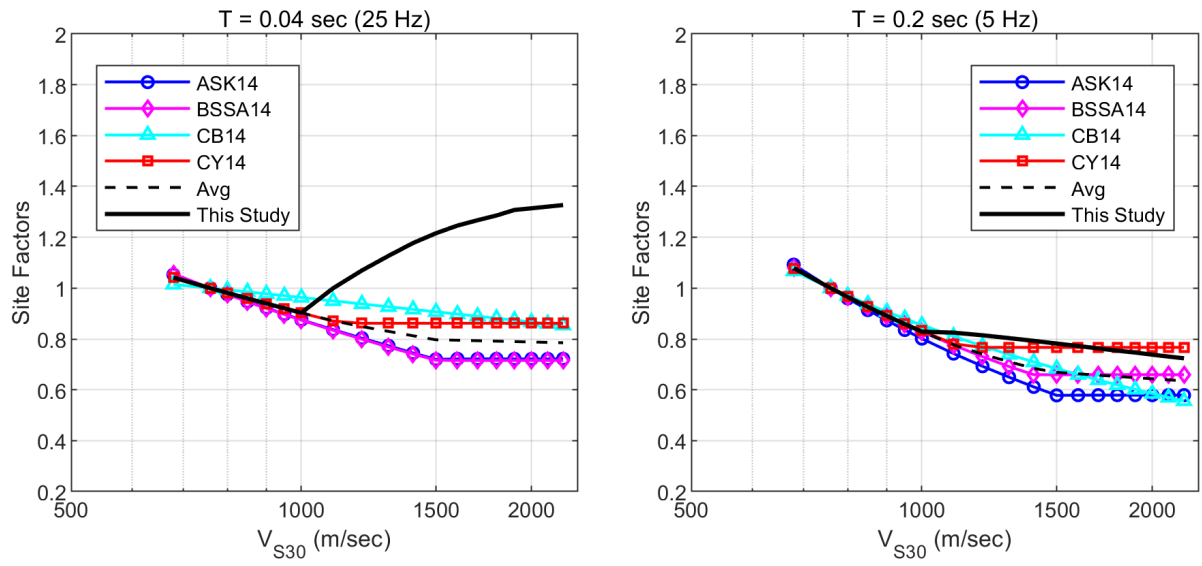

(a)

(b)
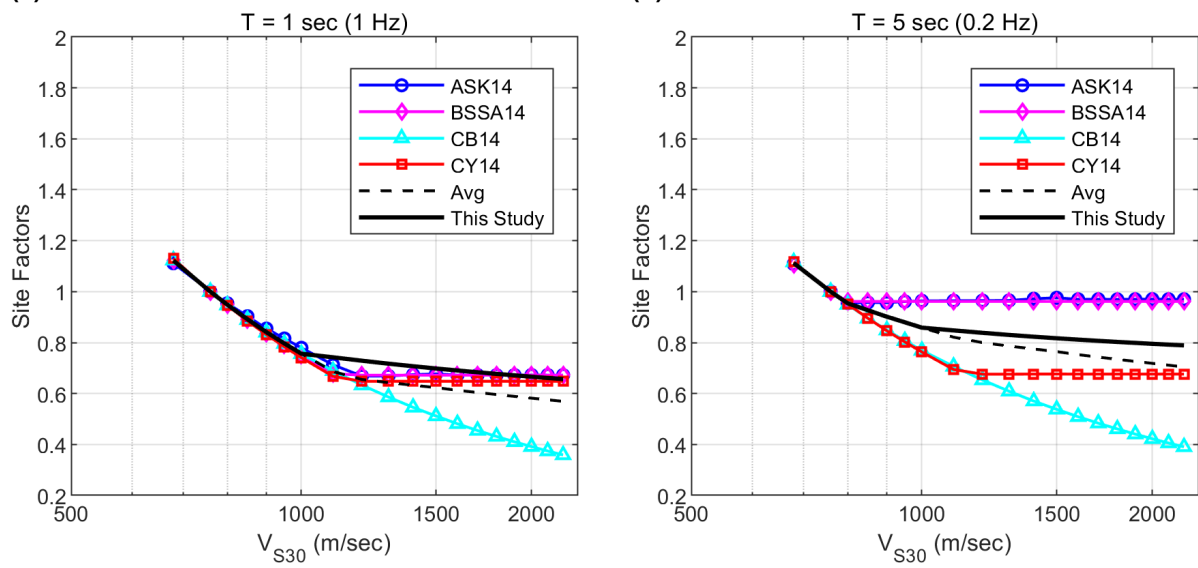

(c)

(d)

Figure 15. Linear $V_{S}$ scaling factors relative to $V_{S 30}$ of $760 \mathrm{~m} / \mathrm{sec}$ for the NGA-West2 GMPEs extrapolated to hard-rock conditions compared to hard-rock scaling factors from this study for frequencies of $25 \mathrm{~Hz}$ (a), $5 \mathrm{~Hz}$ (b), $1 \mathrm{~Hz}$ (c), and $0.2 \mathrm{~Hz}$ (d). 


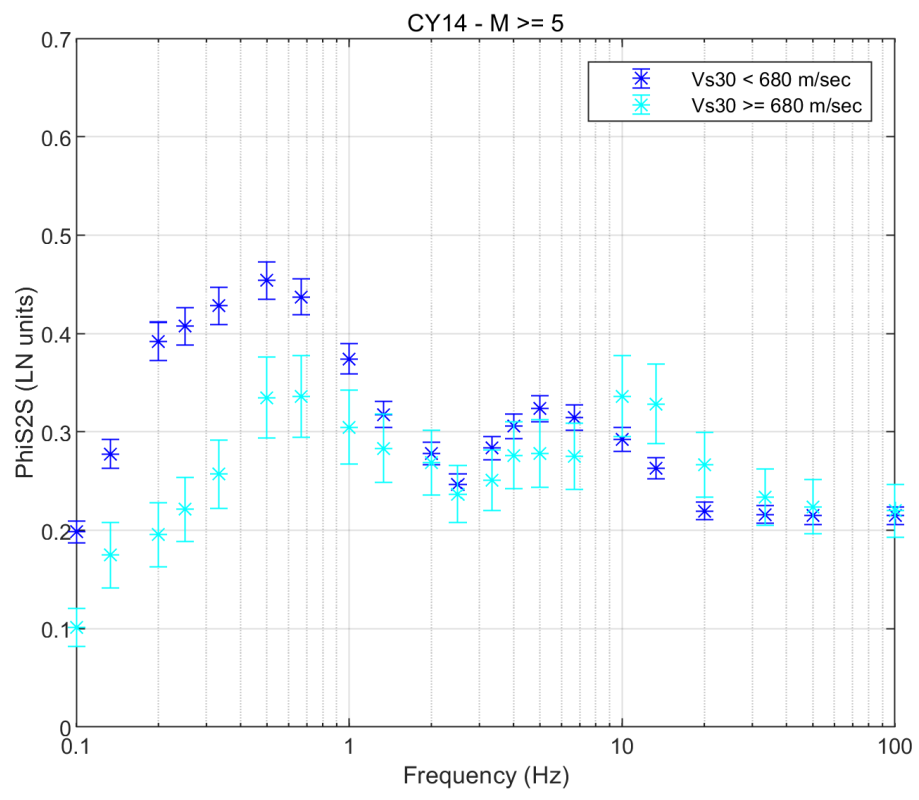

Figure 16. Site-to-site uncertainty $\left(\phi_{S 2 S}\right)$ of CY14 for soil sites with $V_{S 30}<680 \mathrm{~m} / \mathrm{sec}$ and rock sites with $V_{S 30} \geq 680 \mathrm{~m} / \mathrm{sec}$ using data with magnitude $\geq$ to 5 . Error bars show one standard error around the $\phi_{S 2 S}$ estimates.

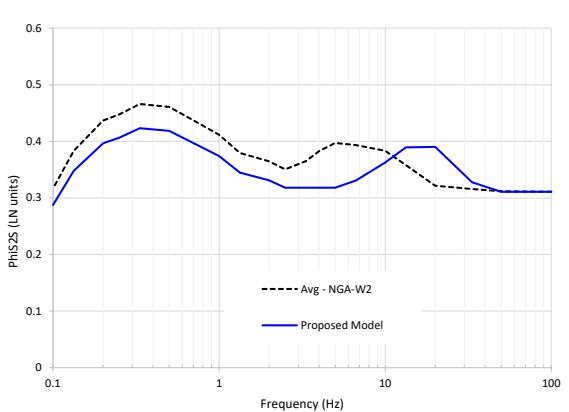

(a)

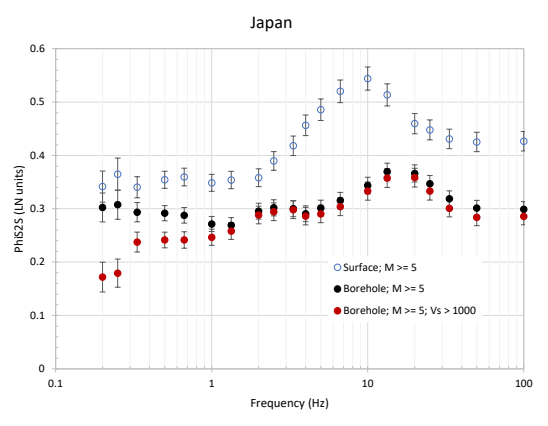

(b)

Figure 17. (a) Average $\phi_{S 2 S}$ based on the NGA-West2 residuals and proposed $\phi_{S 2 S}$ model for hard-rock sites adjusted at high frequencies (b) $\phi_{S 2 S}$ for the Japanese surface and borehole data.

Prepared using sagej.cls 